\title{
Trajectories of Glycemic Control with Clinical Pharmacy Specialist Management of Veterans with Type 2 Diabetes
}

\author{
By \\ (C) 2019 \\ Ted Grabarczyk \\ Pharm.D., University of Wisconsin - Madison, 2010
}

Submitted to the graduate degree program in Clinical Research and the Graduate Faculty of the University of Kansas in partial fulfillment of the requirements for the degree of Master of Science.

Committee Chair: Jonathan Mahnken, $\mathrm{PhD}$

Andrew Roberts, PharmD, PhD

Dennis Grauer, $\mathrm{PhD}$

Date Defended: 25 November 2019 
The thesis committee for Ted Grabarczyk certifies that this is the approved version of the following thesis:

\section{Trajectories of Glycemic Control with Clinical Pharmacy Specialist Management of Veterans with Type 2 Diabetes}

Chair: Jonathan Mahnken, PhD

Graduate Director: Jonathan Mahnken, PhD

Date Approved: 4 December 2019 


\begin{abstract}

\section{Purpose:}

Prior evidence suggests that early, improved control of glycemic control likely lowers the risk of some combination of microvascular and/or macrovascular complications. Furthermore, growing evidence suggests that not only achieving a certain degree of control, but how that control varies also may matter in terms of outcomes. Finally, there is some initial evidence that clinical pharmacy specialist (CPS) services can, on average, lower glycosylated hemoglobin (HbA1c) and improve glycemic control. However, there is insufficient evidence characterizing variation in glycemic control outcomes in patients receiving CPS management of diabetes and factors associated with greater chance of success with that management.
\end{abstract}

\title{
Methods:
}

This is an observational, multicenter, retrospective cohort study of Veterans with type-2 diabetes in Veterans Integrated Service Network 15 managed by clinical pharmacy specialists between 7/1/2013 and 7/1/2017 with a baseline HbA1c level $\geq 8 \%$. Glycosylated hemoglobin measurements were collected for two years following the index date and used to group patients into distinct patterns of $\mathrm{HbA} 1 \mathrm{c}$ trajectories over time using group-based trajectory modeling and posterior probabilities of group membership. Characteristics associated with successful HbA1c trajectories and association of assigned trajectories with all-cause and diabetes-related hospitalizations were analyzed using logistic regression. 


\section{Results:}

A total of 4,119 Veterans were included and successfully divided into six distinct HbA1c trajectory groups: High Gradually Decreasing $(n=325,7.9 \%)$, Moderate Early Decline (n=1692, 41.1\%), Large Early Decline ( $n=231,5.6 \%)$, Uncontrolled Stable ( $n=1468,35.6 \%)$, Early Decline / Subsequent Increase $(n=266,6.5 \%)$, and Very Uncontrolled Stable $(n=137,3.3 \%)$. The Large Early Decline, Moderate Early Decline, and High gradually decreasing groups were classified as successful. Successful trajectories were more likely to reach a target HbA1c of $\leq$ $7 \%$, have shorter duration of pharmacist management, greater utilization of nutrition clinic services. The distinguishing factor between successful and less successful trajectories appears to be the progress made within the first six months of pharmacist management.

\section{Conclusion:}

Patients managed for diabetes can be grouped into distinct patterns of change in glycemic control over time. The first six months of the clinical pharmacist/patient relationship may be important in determining a patient's overall success. Future research is needed to identify pharmacist interventions that increase the likelihood of achieving successful glycemic control trajectory patterns. 


\section{Acknowledgements}

I would like to acknowledge the continued support of my wife and partner. She is inspiring to me not only personally, but has also inspired in me the interest to conduct research that helps improve public health.

I also would like to acknowledge the support and mentorship of my mentors on my thesis committee, Drs. Mahnken, Roberts, and Grauer. Your experience has helped me grow as a clinical researcher.

Additionally, I would like to acknowledge the support of the Department of Veteran's Affairs (VA) and the VA Eastern Kansas Healthcare system research team for their continued commitment to using research to advance the quality of healthcare within the VA.

Finally, and most importantly, I would like to acknowledge the service of our Veterans who served our nation and whose data make this research possible. 


\section{Table of Contents}

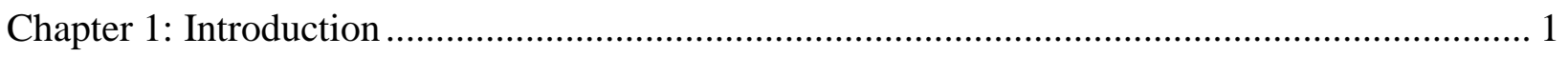

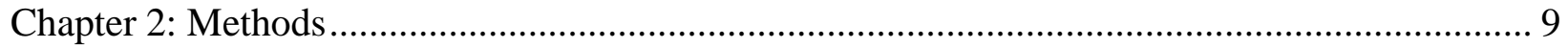

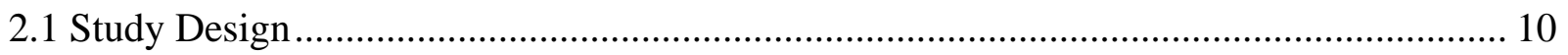

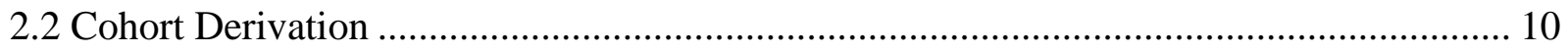

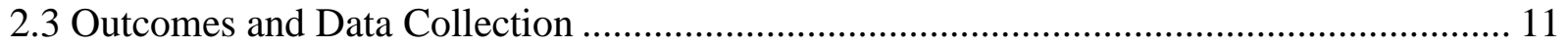

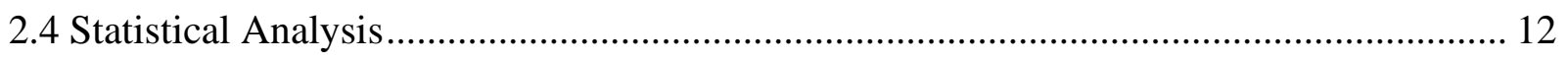

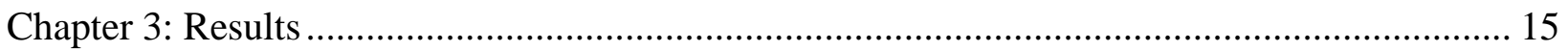

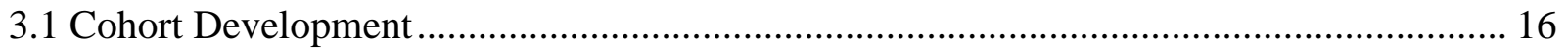

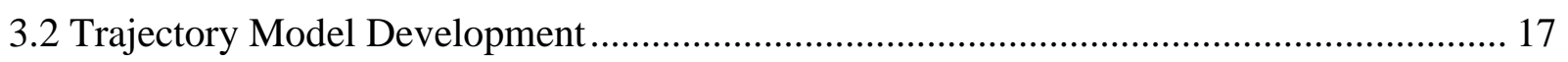

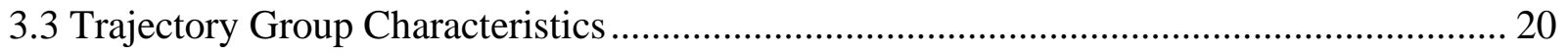

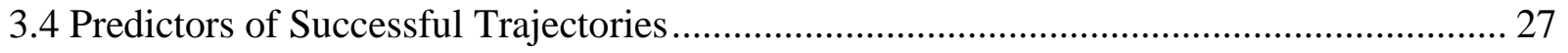

3.5 Relationship to Hospital Admissions ...................................................................... 29

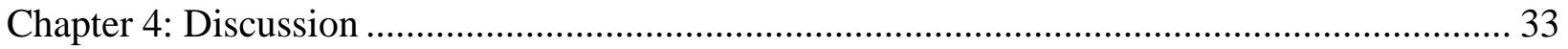

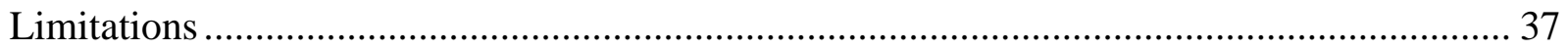

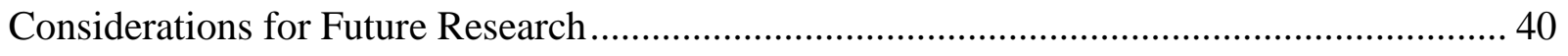

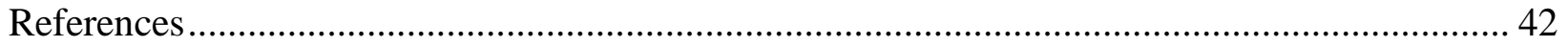

Appendix A: Model Selection Procedure ................................................................... 44

Appendix B: Trajectory Model Diagnostics ............................................................... 46

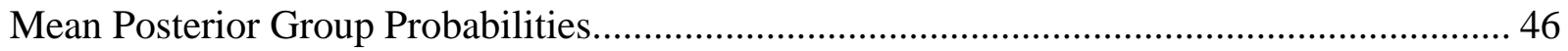

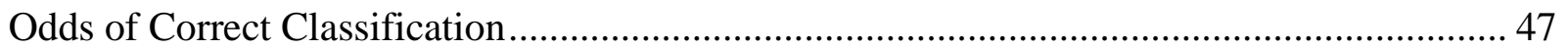

Ratio of Estimated Group Probabilities to the Proportion Assigned to Each Group.............. 47

Reasonableness Check for Glycemic Trajectory Group Assignments ................................. 47

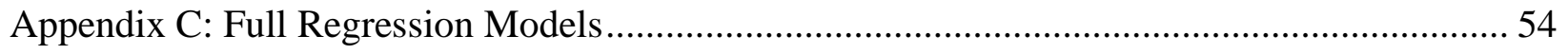

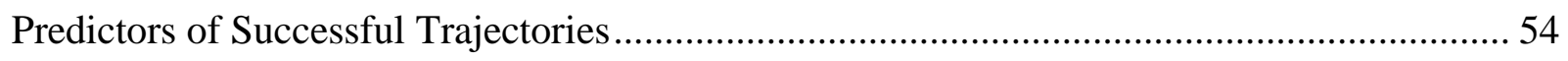

Predictors of All-cause Hospital Admission................................................................ 55

Predictors of Diabetes-related Hospital Admission ....................................................... 56 


\section{List of Figures}

Figure 1: Cohort Derivation and Exclusions .................................................................. 16

Figure 2: Distinct Groups from Group-based Trajectory Modeling Process............................ 17

Figure 3: Trajectory group changes in HbA1c over time ................................................. 18

Figure 4: High Gradually Decreasing Example Trajectories.............................................. 48

Figure 5: Moderate Early Decline Example Trajectories .......................................................... 49

Figure 6: Large Early Decline Example Trajectories ........................................................ 50

Figure 7: Uncontrolled Stable Example Trajectories........................................................ 51

Figure 8: Early Decline / Subsequent Increase Example Trajectories.................................... 52

Figure 9: Very Uncontrolled Stable Example Trajectories................................................ 53

\section{List of Tables}

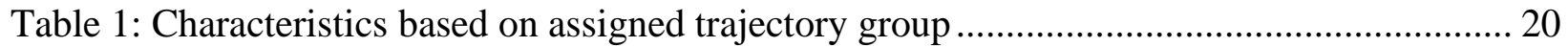

Table 2: Characteristics based on successful vs unsuccessful trajectory groups....................... 23

Table 3: Change in HbA1c percentage points per study time interval ....................................... 26

Table 4: Predictors of Successful Trajectories..................................................................... 27

Table 5: Predictors of all-cause hospital admission................................................................ 30

Table 6: Predictors of diabetes-related hospital admission................................................ 31

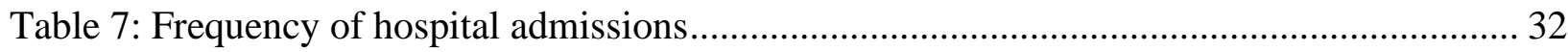

Table 8: Available HbA1c Values at Each Time Interval .................................................. 37

Table 9: Model selection procedure .................................................................................. 44

Table 10: Trajectory group parameter estimates .......................................................... 45

Table 11: Mean posterior group probabilities................................................................ 46

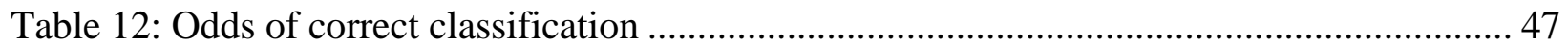

Table 13: Ratio of estimated group probabilities to proportion assigned to each group ............. 47

Table 14: Full model predictors of successful trajectories ..................................................... 54

Table 15: Full model predictors of all-cause hospital admission ............................................ 55

Table 16: Full model predictors of diabetes-related hospital admission .................................. 56 
Chapter 1: Introduction 
Diabetes is a chronic illness that continues to be a serious public health problem, particularly in the Department of Veterans Affairs (VA), with an estimated prevalence among Veterans of $20.5 \%$ in $2013-2014$ as compared to $15.5 \%$ in $2005-2006 .{ }^{1}$ Also, according to $2010-2014$ NHANES data, approximately $49 \%$ of patients nationally with diabetes are not meeting the generally recommended target for glycemic control of a hemoglobin A1c $<7 \%$. $^{2}$ Controlling glycemic status in diabetes is an important measure of quality of primary care services. Keeping HbA1c at least $<9 \%$ is a mainstay in the Healthcare Effectiveness Data and Information Set (HEDIS) measures. ${ }^{3}$ HEDIS is a set of outpatient care performance metrics utilized by many health plans including Medicare. The VA system has adopted a quality measurement system for its medical centers called Strategic Analytics for Improvement and Learning (SAIL) to rate the quality of VA medical centers. ${ }^{4}$ HEDIS measures make up an important component of the SAIL framework as well as including limiting admissions related to ambulatory care sensitive conditions, which are conditions that, if managed well as an outpatient, for instance diabetes, should not result in hospital admission.

Numerous studies have documented the benefits of glycemic control in diabetes. In 1993, the Diabetes Control and Complications Trial (DCCT) showed intensive glycemic control (achieved HbA1c of approximately $7 \%$ versus about $9 \%$ ) in type-1 diabetes delayed the onset and progression of microvascular diabetes complications of retinopathy, nephropathy, and neuropathy. ${ }^{5}$ Interestingly, the Epidemiology of Diabetes Interventions and Complications study (EDIC) extended follow-up of patients in the DCCT cohort for up to 8-14 additional years and found sustained benefit in delayed development of microalbuminuria and reduced rates of neuropathy despite not maintaining intensive glycemic control. ${ }^{6,7}$ This is suggestive that the 
pattern of diabetes control (e.g. early intensive control) may also matter rather than simply overall average achieved level of glycemic control. Similar results were seen in 1998 with the United Kingdom Prospective Diabetes Study (UKPDS) in terms of reduced risk of microvascular complications with intensive glycemic control (HgbA1c $7 \%$ on average) versus less intensive control (approximately $8 \%$ on average) over 10 years in type-2 diabetes. ${ }^{8,9}$

Conversely, subsequent to these early trials, major RCTs showed some more nuanced results. Specifically, the ADVANCE trial showed modest improvement primarily in the rate of nephropathy at the cost of increased rates of severe hypoglycemia. ${ }^{10}$ However, the ACCORD and VA Diabetes Trial (VADT) studies failed to show meaningful improvements in macrovascular or microvascular outcomes with "intensive" versus "standard" therapy. ${ }^{11,12}$ However, there are major differences between the DCCT/EDIC/UKPDS studies as compared to ADVANCE/ACCORD/VADT. Specifically, in the more recent ADVANCE/ACCORD/VADT studies, the study population was older and have had diabetes for a longer period of time prior to study enrollment. However, most importantly, in these subsequent analyses those in the "standard therapy" arms in these more recent trials achieved lower HbA1c of approximately 78\% rather than 7.9-9\% in DCCT and UKPDS. ${ }^{8-12}$ Additionally, higher rates of severe hypoglycemia were observed in the intensive therapy groups of ACCORD/ADVANCE with achieved average $\mathrm{HbA} 1 \mathrm{c}$ in those groups of approximately $6.5 \% .{ }^{10-11}$

Consequently, the 2019 American Diabetes Association Standards of Medical Care in Diabetes recommends targeting an $\mathrm{HbA1c}$ level of $<7 \%$ for most individuals, but possibly adjusting this 
goal to $<8 \%$ for those patients in more challenging situations such as with a history of severe hypoglycemia or advanced diabetes complications. ${ }^{13}$

While general HbA1c targets are now well established, instead of viewing diabetes control as a single measurement at one time point a logical question to ask would be, does the pattern of glycemic control/HbA1c over time matter? An emerging area of research is developing through utilization of group-based trajectory modeling which is an approach used to visualize distinct groupings of patterns of how an outcome (e.g. HbA1c) changes over time and effects of those patterns on outcomes. ${ }^{14-18}$ A retrospective analysis was completed utilizing the Kaiser Permanente Northern California Diabetes Registry to analyze the association of ten-year HbA1c trajectories on diabetes complications and mortality. ${ }^{16}$ The authors fit the data with five distinct trajectory groups of HbA1c control. All HbA1c trajectory groups were associated with higher hazard of microvascular complications as compared to the "low stable" trajectory group which had a relatively stable trajectory of A1c between 7-7.5\%. Also, the 'High decreasing early' trajectory showed an increased mortality risk as compared to the 'low-stable' group after controlling for mean 10-year achieved HbA1c. An earlier study in a Veteran population modeled trajectories of $\mathrm{HbA} 1 \mathrm{c}$ through modeling with use of a time-varying covariate for $\mathrm{HbA} 1 \mathrm{c}$ and found that baseline $\mathrm{HbA1c}$ level was associated with greater risk of mortality and suggested that the slope of the trajectory HbA1c may also be associated with difference in mortality. ${ }^{17}$ Another analysis suggested that those with a 'moderate-increasing' trajectory of $\mathrm{HbAlc}$ is associated with more progression of chronic kidney disease as compared to those classified as 'near-optimal stable'. ${ }^{18}$ Another recent study conducted in Singapore, classified patients with type 2 diabetes into four distinct trajectories of A1c and suggested that those with less stable trajectories (E.g. 
'moderate-increase' and 'high-decrease' subgroups) had an increased rate of stroke, end stage renal disease, and death as compared to the 'low-stable' trajectory. ${ }^{19}$ Finally, while attaining glycemic control is typically sought to prevent traditional microvascular and macrovascular complications of diabetes, another study investigated trajectories of $\mathrm{HbA} 1 \mathrm{c}$ control over time with cognitive outcomes in the elderly, providing some evidence to suggest that non-stable trajectories of HbA1c may be associated with adverse impacts on cognitive performance. ${ }^{20}$

Clinical pharmacy specialists (CPS) are becoming increasingly utilized as part of primary care teams to provide chronic disease management services. VA CPSs are credentialed prescribers as members of the medical staff. They are required to have appropriate baseline credentials and experience to justify their advanced scope of practice. This often occurs through specialized post-graduate training, for example in ambulatory care. A published systematic review by VA Health Services Research and Development summarized current evidence on the outcomes of CPS services, including those related to management of diabetes. ${ }^{21}$ The authors noted a common outcome in these studies was "Goal Attainment", meaning the effectiveness at achieving guideline recommended targets for control of chronic illnesses (e.g. diabetes/hypertension, etc.). In combined results of 6 randomized clinical trials (RCT), pharmacist-led care improved goalattainment for diabetes compared to usual care (RR: 1.83, 95\% CI: $1.44-2.33$ ). However, studies examining the benefits of CPSs on other outcomes were of lower quality.

Recently, some analyses have shown clinical benefit to CPS management of diabetes in the VA. One analysis was conducted at the Memphis VA by retrospective review of 62 CPS managed patients with baseline $\mathrm{HbA} 1 \mathrm{c} \geq 8 \%$ compared to 62 propensity-score matched controls managed 
solely by primary care providers (PCP) between 2012 and $2014 .{ }^{22}$ They were able to show a significant reduction in $\mathrm{HbA} 1 \mathrm{c}$ in the CPS group of approximately 1.5 percentage points from baseline over the course of approximately 14 months as compared to solely PCP management. Another study was conducted at the Cincinnati VA that showed a significant reduction in $\mathrm{HbA1c}$ of an average of $2.2 \%$ from baseline over an average of 18 months with CPS diabetes management. ${ }^{23}$ Though this was a pre/post analysis and did not include a standard of care comparator group. Additionally, another retrospective analysis was conducted in a non-VA internal medicine setting where 82 patients were evaluated against themselves as their own controls comparing a "usual care" run-in phase, to a subsequent pharmacotherapy clinic management phase showing an average reduction of $1.6 \%$ in $\mathrm{HbA} 1 \mathrm{c}$ in the intervention phase compared to usual care. ${ }^{24}$ Finally, a study by Maeng et al demonstrated a reduction in all-cause hospitalization rate $(-19.2 \%, \mathrm{p}=0.02)$, lower medical costs $(-13 \%, \mathrm{p}=0.027)$, more primary care provider visits $(18.5 \%, \mathrm{p}<0.001)$, and a greater proportion of patients attaining goal HbAlc ( $57 \%$ vs. $51 \%, \mathrm{p}<0.0001)$ compared to a cohort not enrolled in pharmacist diabetes management. ${ }^{25}$ Finally, a recent nationwide retrospective cohort study of the effectiveness of VA CPSs in managing uncontrolled diabetes in 53 VA medical centers with over 12,000 patients and propensity-score matched controls showed that CPS providers managed diabetes as well as primary care providers. ${ }^{26}$

While clinical pharmacist management of diabetes can likely assist with goal attainment, due to a limited number of available trained pharmacy specialists, from a practical perspective it is difficult to ascertain which patients are likely to benefit from pharmacist management versus not. This is an important question to investigate in order to target resources most effectively, as there 
are a limited number of clinical pharmacy specialist providers as compared to the total population of patients with diabetes. There is currently limited evidence investigating this question on factors associated with successful results from pharmacist management of diabetes. Lam et al. attempted to address this question by dividing a cohort of pharmacist managed patients into those classified either as clinical success $(\mathrm{HbA} 1 \mathrm{c}$ reduction $\geq 2 \%$ or final $\mathrm{HbA} 1 \mathrm{c}<$ $8 \%$ ) or alternatively clinical failure. ${ }^{27}$ They classified $44.7 \%$ of their population as clinical successes, showing variability in effectiveness. They attempted to identify factors associated with either success or failure. They identified history of cerebrovascular accident (CVA) and higher baseline HbA1c as predictors of success and use of short-acting insulin or a greater number of classes of diabetes medications used as predictors of failure. These results are challenging to interpret, there is no plausible rationale why a history of CVA would be more likely to have success with pharmacy management, thus this may be related to a confounding factor, such as increased healthcare utilization. Conversely, these results would indicate lack of success with more challenging diabetes patients prescribed multiple classes of medications, however, with a lack of a comparator group not utilizing pharmacy services, this could be measuring a "more difficult to manage" patient that other types of providers may also have less overall success with managing.

Overall, in general, improved glycemic control likely helps prevent microvascular and macrovascular complications, though there is a growing body of evidence suggesting that additional factors such as how glycemic control changes over time may play an important role. There is also some initial, encouraging evidence that clinical pharmacy specialists can play a role in improving measures of diabetes control. However, there is very limited evidence 
characterizing how clinical measures change over time with CPS management of diabetes and factors associated with a greater chance of successful improvement in HbA1c with pharmacist management of diabetes. 
Chapter 2: Methods 


\subsection{Study Design}

This is a retrospective, multi-site, cohort study of Veterans with type 2 diabetes mellitus (T2DM) who have received disease management services for T2DM within Veterans Integrated Service Network (VISN) 15 which consists of eight VA medical centers across three midwestern states including Kansas, Missouri, and Illinois. This study was approved by the Kansas City VA Institutional Review Board and the VA Eastern Kansas Healthcare System Research and Development Committee.

The study population was identified using the national Corporate Data Warehouse (CDW) database (VA 79 FR 4377) using resources and facilities at the VA Informatics and Computing Infrastructure (VINCI), (VA HSR RES 13-457). ${ }^{28}$

\subsection{Cohort Derivation}

Patients were identified by use of a flag set through use of an electronic health factor set in the medical record indicating that the patient had seen a pharmacist for management of T2DM between July 1, 2013 and July 1, 2017. The date of the patient's first appointment with a pharmacist for management of diabetes during this interval was considered the index date.

Patients were excluded if their baseline glycosylated hemoglobin (HbA1c) nearest to the index date (between six months prior and one month after) was $<8 \%$ or if a baseline HbA1c could not be identified, if they had any documented visits with a pharmacist for diabetes management in 
the one-year prior to the index date, or if they did not have at least two subsequent visits with a pharmacist for diabetes management following the index date. Veterans with a baseline HbA1c < $8 \%$ were excluded as some Veterans may be given slightly higher $\mathrm{HbA1c}$ goals due to comorbidities and extent of diabetes related complications as consistent with current guideline recommendations. $^{13}$

\subsection{Outcomes and Data Collection}

The primary outcome was change in HbA1c over two-years in a cohort of patients managed by clinical pharmacy specialists for T2DM through classifying patient HbA1c trends into distinct trajectories. Secondary outcomes were to evaluate factors associated with a successful trajectory of change in HgbAlc versus unsuccessful, with success defined as a sustained decrease in HbA1c over the follow-up period. The proportion of patients achieving a goal $\mathrm{HbA} 1 \mathrm{c}$ of $\leq 7 \%$ at some point during the second of the two-year follow-up period was determined. Finally, as an exploratory, hypothesis-generating analysis, occurrence of all-cause and diabetes-related hospitalization and association with glycemic control trajectory groups was determined. Patients were followed retrospectively for the entire two-year period, even if clinical pharmacy specialist management of diabetes had ceased.

Data were collected from the electronic health record utilizing Microsoft SQL Server query. Demographic and clinical data collected at baseline included sex, age, race, weight (within 6 months prior or 3 months after the index date), blood pressure (within 90 days of the index date), co-morbidities (within 2 years prior to the index date), baseline laboratory measurements within 
1-year prior or 90 days after the index date (serum creatinine, low density lipoprotein, high density lipoprotein, triglycerides, and total cholesterol), and hypoglycemic medications (within 6 months prior to the index date). Additional data collected during the two-year post-index study period included HbA1c at 3-month intervals, hypoglycemic medication dispensed within the year following the last pharmacist visit, blood pressure and lipid profile as of the last pharmacist visit, diagnosis of alcohol or substance use disorders, nutrition or weight management visits, participation in the home telehealth program, number of pharmacy visits for diabetes management, and the proportion of those visits that were conducted via telephone or via clinical video telehealth technology.

Diagnoses for co-morbidities were determined from ICD9/ICD10 coding from electronic medical record problem lists, inpatient admissions, and outpatient visits. For each 3-month interval, the closest $\mathrm{HbA} 1 \mathrm{c}$ reading to the appropriate time interval from the index date (e.g. 3 months, 6 months, 9 months, etc.) was taken between two months prior and 1 month following that date ensuring the same $\mathrm{HbA1c}$ reading is not used for more than one interval.

\subsection{Statistical Analysis}

All analyses were performed using SAS Enterprise Guide Version X (SAS, Cary, NC, USA) within the VINCI environment. The VINCI environment is a secure research environment hosted on centralized VA servers. Group-based trajectory modeling using PROC TRAJ in SAS was utilized using a censored normal model to group patients into defined and distinct trajectories of change in HbA1c over the study period. ${ }^{29,30}$ As suggested by prior literature, the number of 
trajectory groups to include in the model was determined based on selecting the number of groups that minimized the Bayesian Information Criterion (BIC) up to a maximum total number of groups with each group initially modeled as a quadratic-order equation or one zero-order equation and the rest quadratic order if it is thought that one group may have a trajectory that does not change with time. ${ }^{31} \mathrm{~A}$ maximum of six groups was chosen as a larger number of groups would be difficult to clinically interpret. Subsequently, each group was tested in order by adjusting the shape (or order of the polynomial) of each trajectory group to minimize the total model BIC. Terms were added to the model for each group until the BIC began to increase. Two times the change in BIC between the prior model and the more complicated model was also used to evaluate the benefit of including more terms in the model where larger negative values indicated greater support for keeping the more complicated model. ${ }^{32}$ After trajectories were determined, patients were assigned to a single trajectory group by SAS based on posterior probabilities of group membership from the output of the PROC TRAJ procedure. ${ }^{31}$ Trajectory groups were then categorized as either "successful" or "unsuccessful" based on visual inspection with "successful" groups illustrating a sustained reduction in HbA1c. Subsequently, logistic regression was utilized to evaluate for characteristics associated with successful versus unsuccessful trajectory groups. Variables included in the final regression model were determined through backwards elimination with a p-value to stay in the model of 0.1 . The final model was evaluated with the Hosmer and Lemeshow Goodness-of-fit test and by comparing the results of the reduced model with the full model including all predictors.

Logistic regression was also utilized to evaluate the association of glycemic control trajectories and all-cause or diabetes-related acute hospitalization. Acute hospitalization within the VA CDW 
was determined based on a list of treating specialties published by Vincent et al. ${ }^{33}$ Model selection and evaluation was performed as stated above; however, the assigned glycemic control trajectory group was included in the model regardless of significance as this was the primary predictor of interest.

Baseline and study period characteristics across trajectory groups were analyzed by Pearson $\chi^{2}$ test for categorical variables and ANOVA for continuous variables. For continuous data, normality of the data was assessed through inspection of the QQ plot and equality of variances was assessed through inspection of a plot of predicted versus residual values for any obvious patterns. If the data showed obvious deviations from normality, the Kruskal-Wallis test was used. Characteristics by success or not were analyzed by Pearson $\chi^{2}$ test for categorical variables and student t-test for continuous variables. For continuous measures, if normality could not be assumed, the Wilcoxon Rank Sum test was used. Equality of variances was assessed using the folded F statistic. If variances could not be assumed to equal, the Satterthwaite method was used instead of pooled variance. The 2 -sided alpha level was set at 0.05 hence, p-values $<0.05$ was considered statistically significant. 
Chapter 3: Results 


\subsection{Cohort Development}

A total of 10,331 Veterans met the study inclusion criteria which comprised the initial cohort. After excluding patients with no appropriate HbA1c measurement at baseline $(n=231)$, a baseline $\mathrm{HbA1c}<8 \%(\mathrm{n}=3,375)$, less than three total pharmacy visits for management of T2DM $(n=2,710)$, or a pharmacy visit for management of T2DM within the year prior to the index date $(\mathrm{n}=1,233)$ a final cohort of 4,119 Veterans was established (Figure 1).

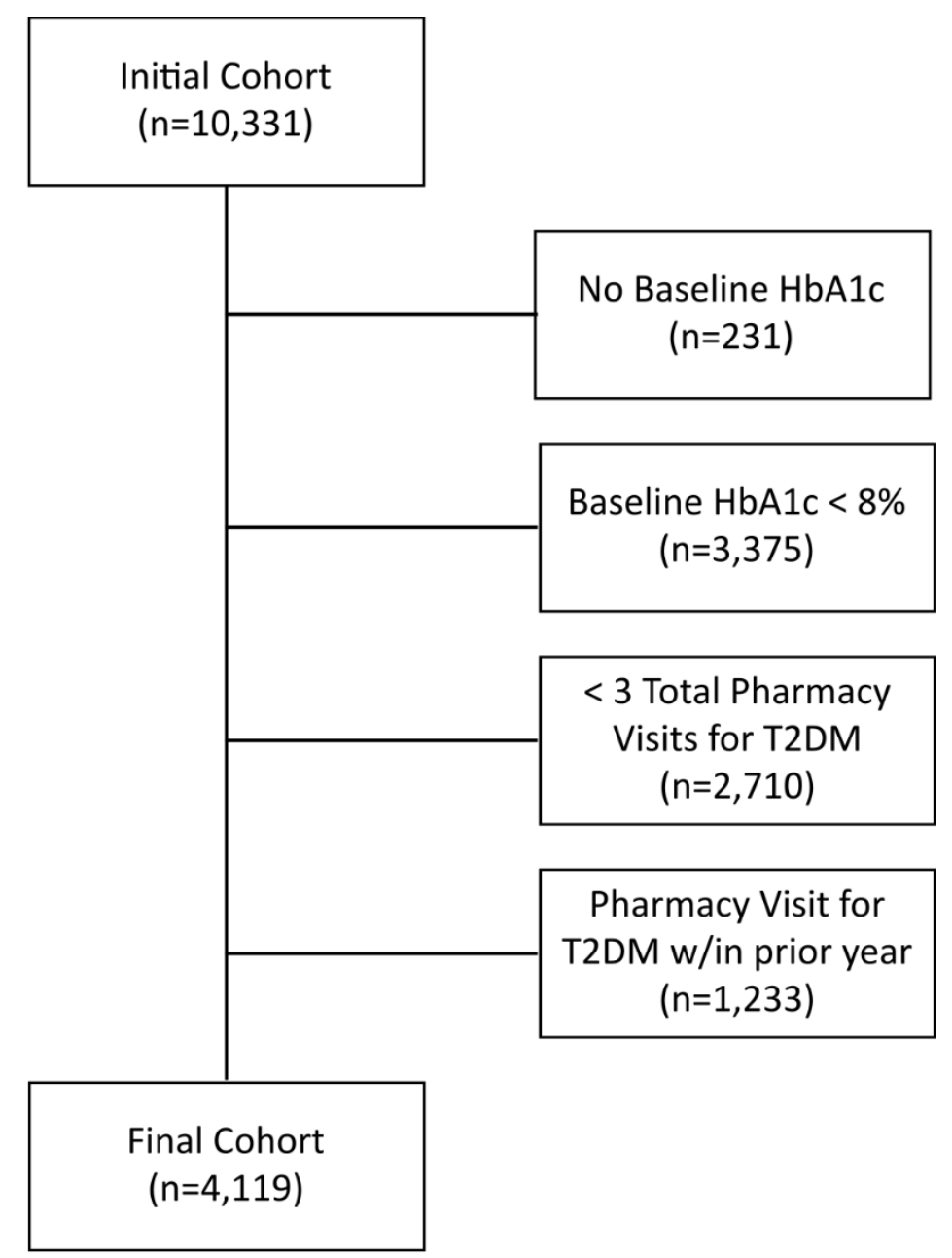

Note: Patients may have met $>1$ exclusion criterion

Figure 1: Cohort Derivation and Exclusions 


\subsection{Trajectory Model Development}

Model selection to determine the optimal fit of trajectory groups to the data was conducted as previously stated through first determining the optimal number of groups up to the maximum of six groups and then determining the best equation order to model each group by minimization of BIC. It was decided to begin with a two-group model including a zero-order group and a quadratic order group, as it was conceivable that there was a group with an essentially unchanged HbA1c throughout the study period. ${ }^{31}$ Further details regarding the steps of determining the final model can be located in Appendix A.

In the final model, patients fell into six distinct trajectory groups of HbA1c over time (Figure 2): High gradually decreasing, moderate early decline, large early decline, uncontrolled stable, early decline/subsequent increase, and very uncontrolled stable.

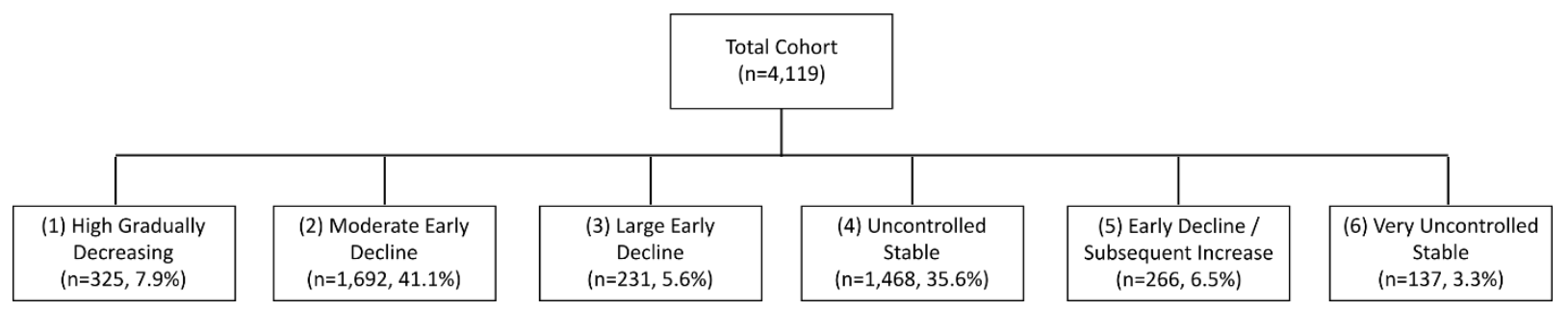

Figure 2: Distinct Groups from Group-based Trajectory Modeling Process 


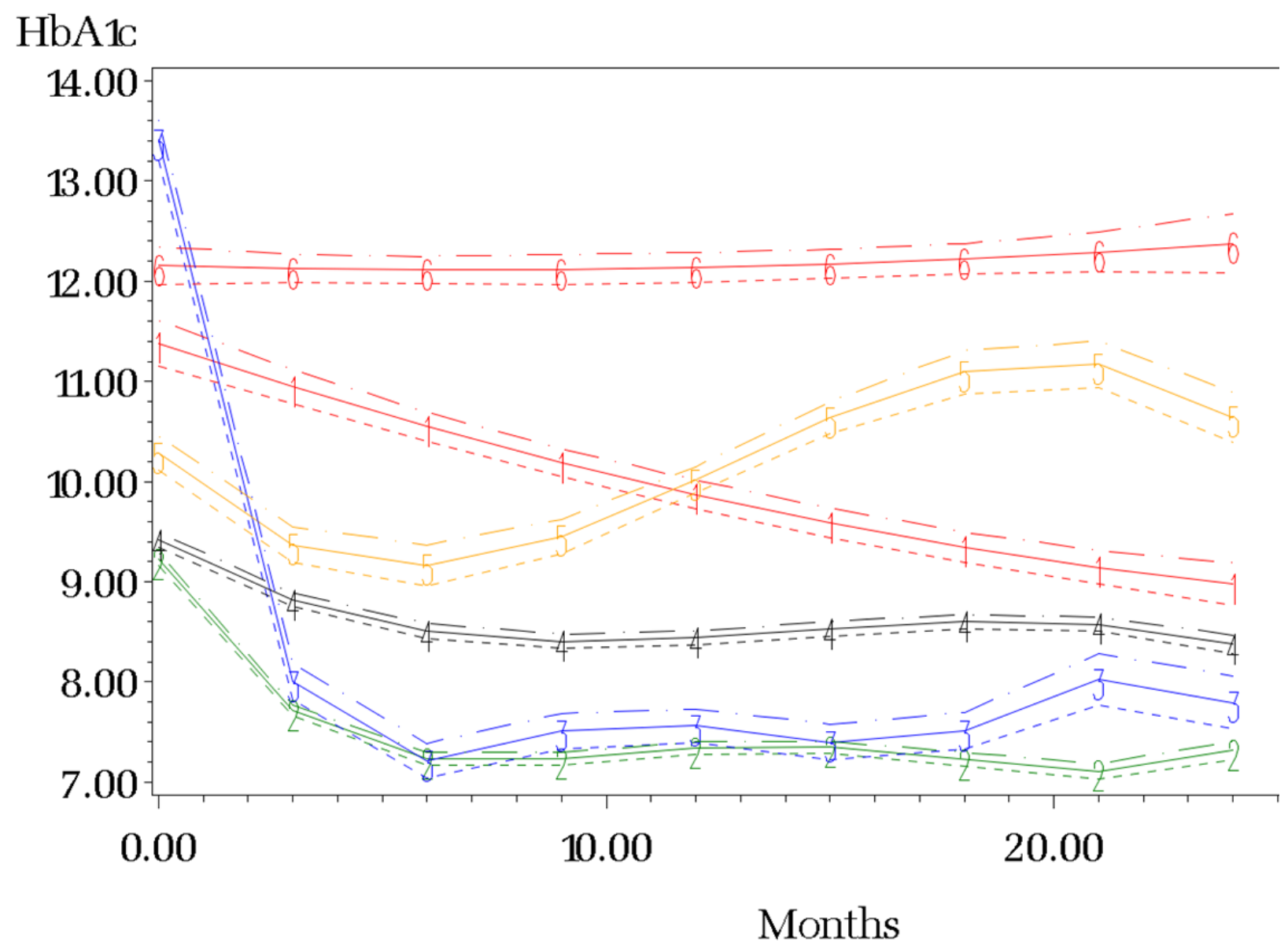

\section{Legend:}

$1=$ High Gradually Decreasing $(n=325,7.9 \%), 2$ = Moderate Early Decline $(n=1692,41.1 \%)$

$3=$ Large Early Decline $(\mathrm{n}=231,5.6 \%), 4=$ Uncontrolled Stable $(\mathrm{n}=1468,35.6 \%)$

$5=$ Early Decline/Subsequent Increase $(n=266,6.5 \%), 6=$ Very Uncontrolled Stable $(n=137,3.3 \%)$

Figure 3: Trajectory group changes in HbA1c over time

In order to determine model adequacy, several analyses were conducted to check the model.

Firstly, the average of the posterior group probabilities of subjects assigned to each group were determined for each group. Ideally, the average posterior probability for the assigned group would be 1 , however, average posterior probabilities above 0.7 indicate acceptable fit to the model. ${ }^{31}$ All group average posterior probabilities for this model were above 0.8 (Appendix B). The odds of correct classification was also calculated which was above 5 for each group also 
indicating acceptable fit of the data to the model (Appendix B) ${ }^{31}$ Finally, the estimated group probabilities in the population should be similar to the proportion of subjects assigned to each group, leading to a ratio at or near 1 , which was also observed for this sample (Appendix B). ${ }^{31}$ Overall, these data seem to fit reasonably well to these six modeled trajectory groups.

As illustrated from Figure 1, despite patients being classified into six distinct trajectory groups of HbAlc over time, a large majority of patients fell into either the 'Moderate Early Decline' or the 'Uncontrolled Stable' groups (76.7\%). 


\subsection{Trajectory Group Characteristics}

Baseline and study period characteristics of patients assigned to each glycemic control trajectory group can be seen below in Table 1 .

Table 1: Characteristics based on assigned trajectory group

\begin{tabular}{|c|c|c|c|c|c|c|}
\hline & $\begin{array}{l}\text { High Gradually } \\
\text { Decreasing } \\
(n=325)\end{array}$ & $\begin{array}{c}\text { Moderate } \\
\text { Early Decline } \\
\text { (n=1692) }\end{array}$ & $\begin{array}{l}\text { Large Early } \\
\text { Decline } \\
\text { (n=231) }\end{array}$ & $\begin{array}{c}\text { Uncontrolled } \\
\text { Stable } \\
(n=1468)\end{array}$ & $\begin{array}{c}\text { Early Decline / } \\
\text { Subsequent } \\
\text { Increase } \\
(\mathrm{n}=\mathbf{2 6 6})\end{array}$ & $\begin{array}{c}\text { Very } \\
\text { Uncontrolled } \\
\text { Stable } \\
(n=137) \\
\end{array}$ \\
\hline Age, yrs (SD)* & $60.5(10.3)$ & $65.3(9.4)$ & $62.5(10.5)$ & $65.1(9.8)$ & $60.4(10.5)$ & $57.2(10.1)$ \\
\hline Male, no. (\%) & $312(96)$ & 1641 (97) & $226(97.8)$ & $1421(96.8)$ & $251(94.4)$ & $130(94.9)$ \\
\hline \multicolumn{7}{|l|}{ Race, no. (\%)* } \\
\hline White & $216(66.5)$ & $1323(78.2)$ & $150(64.9)$ & $1151(78.4)$ & $192(72.2)$ & $76(55.5)$ \\
\hline Black or African American & $90(27.7)$ & $296(17.5)$ & $66(28.6)$ & $237(16.1)$ & $61(22.9)$ & $53(38.7)$ \\
\hline Other & $19(5.8)$ & $73(4.3)$ & $15(6.5)$ & $80(5.5)$ & $13(4.9)$ & $8(5.8)$ \\
\hline $\mathrm{BMI}, \mathrm{kg} / \mathrm{m} 2(\mathrm{SD})^{0}$ & $33.9(7.2)$ & $34.1(6.6)$ & $32.4(6.0)$ & $34.2(6.7)$ & $33.9(6.5)$ & $32.9(6.6)$ \\
\hline Pharmacy Visits, no. (SD) ${ }^{0}$ & $10.6(8.7)$ & $10.1(7.2)$ & $10.2(8.4)$ & $12.1(8.7)$ & $9.5(7.7)$ & $7.3(5.2)$ \\
\hline Telephone Visit, \%, (SD) & $9.8(23)$ & $13.4(26.9)$ & $12.6(25.4)$ & $13.3(26.2)$ & $9.4(22)$ & $12.7(23.1)$ \\
\hline Clinical Video Telehealth, \% (SD) & $1.6(9.9)$ & $0.97(8.5)$ & $1.6(10.1)$ & $1.5(10.8)$ & $0.8(8.1)$ & $1.5(10)$ \\
\hline Home Telehealth, \%* & $85(26.2)$ & $314(18.6)$ & $41(17.8)$ & $376(25.6)$ & $71(26.7)$ & $30(21.9)$ \\
\hline \multicolumn{7}{|l|}{ Nutrition/Weight Mgmt, \%* } \\
\hline $0-1$ Visits & $237(72.9)$ & $1322(78.1)$ & $162(70.1)$ & $1123(76.5)$ & $200(75.2)$ & $99(72.3)$ \\
\hline 2-5 Visits & $57(17.5)$ & $239(14.1)$ & $49(21.2)$ & $229(15.6)$ & $50(18.8)$ & $32(23.4)$ \\
\hline $6+$ Visits & $31(9.5)$ & $131(7.7)$ & $20(8.7)$ & $116(7.9)$ & $16(6)$ & $6(4.4)$ \\
\hline \multicolumn{7}{|l|}{ Pharmacy follow-up duration (SD)* } \\
\hline$<6$ Months & $57(17.5)$ & $493(29.1)$ & $86(37.2)$ & $239(16.3)$ & $61(22.9)$ & $42(30.7)$ \\
\hline 6-12 Months & $84(25.9)$ & $442(26.1)$ & $53(22.9)$ & $372(25.3)$ & $95(35.7)$ & $50(36.5)$ \\
\hline$\geq 12$ Months & $184(56.6)$ & $757(44.7)$ & $92(39.8)$ & $857(58.4)$ & $110(41.4)$ & $45(32.9)$ \\
\hline Baseline A1c, \% (SD) ${ }^{\circ}$ & $11.7(1.4)$ & $9.2(1.0)$ & $13.5(1.4)$ & $9.5(1)$ & $10.3(1.4)$ & $12.5(1.7)$ \\
\hline \multicolumn{7}{|l|}{ Creatinine Clearance, \%* } \\
\hline$\geq 60 \mathrm{ml} / \mathrm{min}$ & $279(85.9)$ & $1398(82.6)$ & $192(83.1)$ & $1168(79.6)$ & $229(86.1)$ & $120(87.6)$ \\
\hline $30-59 \mathrm{ml} / \mathrm{min}$ & $35(10.8)$ & $191(11.3)$ & $33(14.3)$ & $185(12.6)$ & 21 (7.9) & $8(5.8)$ \\
\hline$<30 \mathrm{ml} / \mathrm{min}$ & 0 & $23(1.4)$ & $2(0.9)$ & $24(1.6)$ & 0 & $1(0.7)$ \\
\hline Unknown & $11(3.4)$ & $80(4.7)$ & $4(1.7)$ & $91(6.2)$ & $16(6)$ & $8(5.8)$ \\
\hline BP, baseline, $\mathrm{mmHg}(\mathrm{SD})^{\circ}$ & $136 / 79(18 / 12)$ & $133 / 76(17 / 12)$ & $131 / 78(16 / 12)$ & $135 / 76(18 / 12)$ & $134 / 79(17 / 13)$ & $133 / 79(16 / 12)$ \\
\hline BP, final, mmHg (SD) [DBP*] & $134 / 77(19 / 12)$ & $133 / 75(18 / 11)$ & $132 / 77(18 / 11)$ & $133 / 75(18 / 12)$ & $134 / 78(21 / 14)$ & $134 / 81(22 / 14)$ \\
\hline \multicolumn{7}{|l|}{ Lipid levels, mg/dl, baseline: } \\
\hline Total Cholesterol (SD) ${ }^{\diamond}$ & $195.8(69.5)$ & $168.1(51.2)$ & $188(57.1)$ & $168(70.5)$ & $178.4(53.7)$ & $194.5(53.4)$ \\
\hline Triglycerides $(S D)^{\diamond}$ & $298.1(407.3)$ & $227.2(230.2)$ & $276.3(234.9)$ & $214.8(188)$ & $231.9(201.4)$ & $236.6(184.3)$ \\
\hline $\mathrm{HDL}(\mathrm{SD})^{\circ}$ & $38.1(9.7)$ & $36.7(9.8)$ & $36.4(8.7)$ & $36.7(9.9)$ & $37.6(10.2)$ & $39.7(10.3)$ \\
\hline $\operatorname{LDL}(S D)^{\ominus}$ & $106.3(43.2)$ & 91 (34.6) & $105.5(45.5)$ & $91.1(33.7)$ & $98.7(38)$ & $111.7(41.6)$ \\
\hline \multicolumn{7}{|l|}{ Lipid levels, mg/dl, final visit } \\
\hline Total Cholesterol (SD) ${ }^{\diamond}$ & $170.6(48.1)$ & $150.8(42.3)$ & $158(48.6)$ & $155.8(43.2)$ & $170.7(47.4)$ & $193.4(55.9)$ \\
\hline Triglycerides (SD) ${ }^{\circ}$ & $207.3(162.7)$ & $173.6(121.8)$ & $186.9(188.8)$ & $188.3(161.7)$ & $219.6(188.6)$ & $254.6(239.9)$ \\
\hline $\mathrm{HDL}(\mathrm{SD})^{\circ}$ & $37.5(9.8)$ & $36.6(9.2)$ & $36.8(9.4)$ & $36.4(9.3)$ & $37.3(9.9)$ & $39.3(10)$ \\
\hline $\operatorname{LDL}(S D)^{\ominus}$ & 96.6 (39.6) & $82.1(31.3)$ & $87.6(33.6)$ & $85.2(32.7)$ & 94.5 (35.6) & $108.4(43.9)$ \\
\hline Test Strips/day, mean no (SD) & $2.8(1.2)$ & $2.3(1.4)$ & $2.6(1.3)$ & $2.7(1.3)$ & $2.5(1.4)$ & $2.8(1.2)$ \\
\hline
\end{tabular}




\begin{tabular}{|c|c|c|c|c|c|c|}
\hline & $\begin{array}{l}\text { High Gradually } \\
\text { Decreasing } \\
\text { (n= 325) }\end{array}$ & $\begin{array}{c}\text { Moderate } \\
\text { Early Decline } \\
\text { (n=1692) }\end{array}$ & $\begin{array}{l}\text { Large Early } \\
\text { Decline } \\
(n=231)\end{array}$ & $\begin{array}{l}\text { Uncontrolled } \\
\text { Stable } \\
(n=1468)\end{array}$ & $\begin{array}{l}\text { Early Decline / } \\
\text { Subsequent } \\
\text { Increase } \\
(\mathrm{n}=266)\end{array}$ & $\begin{array}{c}\text { Very } \\
\text { Uncontrolled } \\
\text { Stable } \\
(n=137)\end{array}$ \\
\hline \multicolumn{7}{|l|}{ Medications, Baseline } \\
\hline Total, no. (SD) ${ }^{\diamond}$ & $2.03(0.96)$ & $1.94(0.99)$ & $1.81(1.11)$ & $2.14(0.96)$ & $2.08(0.97)$ & $1.93(1.07)$ \\
\hline Metformin, \% & $162(49.9)$ & $958(56.6)$ & $121(52.4)$ & 787 (53.6) & $151(56.8)$ & 65 (47.5) \\
\hline Sulfonylurea, \%* & $104(32)$ & $652(38.5)$ & $67(29)$ & $552(37.6)$ & $90(33.8)$ & 39 (28.5) \\
\hline DPP-4 Inhibitor, \%* & $18(5.5)$ & $151(8.9)$ & $13(5.6)$ & $119(8.1)$ & $16(6)$ & $3(2.2)$ \\
\hline GLP-1 Agonist, \% & $1(0.3)$ & $16(1)$ & $2(0.9)$ & $25(1.7)$ & $3(1.1)$ & $2(1.5)$ \\
\hline SGLT-2 Inhibitor, \% & 0 & $1(0.1)$ & 0 & $2(0.1)$ & 0 & 0 \\
\hline Thiazolidinedione, \% & $12(3.7)$ & $50(3)$ & $8(3.5)$ & $41(2.8)$ & $9(3.4)$ & $2(1.5)$ \\
\hline \multicolumn{7}{|l|}{ Insulin, \%* } \\
\hline Basal/Bolus & $137(42.2)$ & $562(33.2)$ & $74(32)$ & $635(43.3)$ & $113(42.5)$ & $57(41.6)$ \\
\hline Basal Only & $78(24)$ & $316(18.7)$ & $55(23.8)$ & $305(20.8)$ & $50(18.8)$ & 35 (25.6) \\
\hline Prandial Only & $8(2.5)$ & $18(1.1)$ & $5(2.2)$ & $36(2.5)$ & $8(3)$ & $3(2.2)$ \\
\hline$U-500$ & $2(0.6)$ & $2(0.1)$ & 0 & $4(0.3)$ & 0 & $1(0.7)$ \\
\hline Statin, \%* & $212(65.2)$ & 1149 (67.9) & $130(56.3)$ & $1016(69.2)$ & $177(66.5)$ & $77(56.2)$ \\
\hline \multicolumn{7}{|l|}{ Medications, final visit } \\
\hline Total, no. $(S D)^{\diamond}$ & $2.94(0.96)$ & $2.61(0.98)$ & $2.51(0.86)$ & $2.94(0.96)$ & $2.89(0.91)$ & $2.82(0.82)$ \\
\hline Metformin, \%* & $211(64.9)$ & $1206(71.3)$ & $165(71.4)$ & $966(65.8)$ & $171(64.3)$ & 79 (57.7) \\
\hline Sulfonylurea, \%* & $68(20.9)$ & $618(36.5)$ & 73 (31.6) & $443(30.2)$ & $89(33.5)$ & 39 (28.5) \\
\hline DPP-4 Inhibitor, \%* & $46(14.2)$ & $394(23.3)$ & $34(14.7)$ & $343(23.4)$ & $48(18.1)$ & $19(13.4)$ \\
\hline GLP-1 Agonist, \%* & $60(18.5)$ & $241(14.2)$ & $20(8.7)$ & $270(18.4)$ & $41(15.4)$ & $15(11)$ \\
\hline SGLT-2 Inhibitor, \%* & $31(9.5)$ & $86(5.1)$ & $5(2.2)$ & $100(6.8)$ & $17(6.4)$ & $1(0.7)$ \\
\hline Thiazolidinedione, \% & $32(9.9)$ & $102(6)$ & $10(4.3)$ & $98(6.7)$ & $20(7.5)$ & $7(5.1)$ \\
\hline \multicolumn{7}{|l|}{ Insulin, \%* } \\
\hline Basal/Bolus & $216(66.5)$ & $662(39.1)$ & $97(42)$ & $856(58.3)$ & $158(59.4)$ & $102(74.5)$ \\
\hline Basal Only & $62(19.1)$ & $415(24.5)$ & $77(33.3)$ & $345(23.5)$ & $64(24.1)$ & 19 (13.9) \\
\hline Prandial Only & 6 (1.9) & $14(0.8)$ & $2(0.9)$ & $15(1)$ & $4(1.5)$ & $3(2.2)$ \\
\hline U-500 & $2(0.6)$ & $7(0.4)$ & 0 & $8(0.5)$ & 0 & 0 \\
\hline Statin, \% & $276(84.9)$ & $1404(83)$ & $180(77.9)$ & $1205(82.1)$ & $225(84.6)$ & $110(80.3)$ \\
\hline \multicolumn{7}{|l|}{ Comorbid Conditions, \% } \\
\hline Alcohol Use Disorder* & 29 (8.9) & $151(8.9)$ & $23(10)$ & $100(6.8)$ & $28(10.5)$ & $20(14.6)$ \\
\hline Substance Use Disorder* & $76(23.4)$ & $340(20.1)$ & $55(23.8)$ & $263(17.9)$ & $72(27.1)$ & $30(21.9)$ \\
\hline Hypertension* & $278(85.5)$ & $1523(90)$ & $193(83.6)$ & 1344 (91.6) & $238(89.5)$ & $120(87.6)$ \\
\hline Heart Failure* & 41 (12.6) & $176(10.4)$ & $18(7.8)$ & $199(13.6)$ & 29 (10.9) & $17(12.4)$ \\
\hline A1c at goal within second year* & $26(8)$ & $1041(61.5)$ & $101(43.7)$ & $185(12.6)$ & $3(1.1)$ & 0 \\
\hline
\end{tabular}

$*: p<0.05$ (ANOVA), $:$ p $<0.05$ (Kruskal-Wallis)

$B M I=$ Body mass index $B P=$ Blood pressure, $H D L=$ High density lipoproteins, $L D L=$ Low density lipoproteins, DPP-4 = Dipeptidyl peptidase -4 , DPP-4 = Dipeptidyl peptidase-4, GLP-1 = Glucagon-lke peptide 1, SGLT-2 = Sodium-glucose cotransporter 2

Across trajectory groups, patients were relatively similar. Patients were mostly male with average ages of approximately 60 years with BMI $>30 \mathrm{~kg} / \mathrm{m}^{2}$, sufficient renal function, and relatively well controlled blood pressure. As would be expected, HbA1c at baseline was significantly different among glycemic control trajectory groups, with as expected, a majority of patients falling into trajectory groups starting from a modestly uncontrolled HbA1c on average 
between $9-10 \%$. Also as expected, a greater proportion of patients in the Very Uncontrolled group were prescribed basal/bolus insulin at the end of pharmacist follow-up and that the rates of usage of insulin increased in all groups from baseline to the end of pharmacist follow-up.

Interestingly, patients in the 'Large Early Decline' group showed a similar average duration of pharmacist management as the 'Very Uncontrolled Stable' group; however, those in the 'Large Early Decline' group had on average three more visits with a pharmacy provider. The reason for this is uncertain and an area for further inquiry; however, it is possible that early, more frequent contact is helpful.

Finally, there generally was an increase in utilization of most classes of medications for treatment of diabetes (except sulfonylureas) which would be consistent with intensifying diabetes treatment regimens. Additionally, there appeared to be a consistent increase in the utilization of statins from baseline to the end of the 2-year study period or post-pharmacist management period.

The 'Moderate Early Decline', 'Large Early Decline', and the 'High Gradually Decreasing' glycemic control trajectory groups were defined as 'Successful' trajectories $(n=2248,55 \%)$ based on a sustained decrease in $\mathrm{HbA1c}$ over time. Conversely, the remaining three groups were classified as 'Unsuccessful' ( $\mathrm{n}=1871,45 \%)$. 
Baseline and study period characteristics for those trajectories classified as 'Successful' versus 'Unsuccessful' are shown in Table 2.

Table 2: Characteristics based on successful vs unsuccessful trajectory groups

\begin{tabular}{|c|c|c|c|}
\hline & $\begin{array}{l}\text { Unsuccessful } \\
\text { Trajectory } \\
(\mathrm{n}=1871) \\
\end{array}$ & $\begin{array}{l}\text { Successful } \\
\text { Trajectory } \\
(\mathrm{n}=\mathbf{2 2 4 8}) \\
\end{array}$ & P-Value \\
\hline Age, yrs (SD) & $63.9(10.2)$ & $64.3(9.8)$ & 0.189 \\
\hline Male, \% & $1802(96.3)$ & 2179 (96.9) & 0.2721 \\
\hline Race, \% & & & 0.3981 \\
\hline White & $1419(75.8)$ & $1689(75.1)$ & \\
\hline Black or African American & $351(18.8)$ & $452(20.1)$ & \\
\hline Other & $101(5.4)$ & $107(4.8)$ & \\
\hline $\mathrm{BMI}, \mathrm{kg} / \mathrm{m} 2$ (SD) & $34(6.6)$ & $33.9(6.7)$ & 0.5958 \\
\hline Pharmacy Visits, no. (SD) ${ }^{\circ}$ & $11.4(8.5)$ & $10.1(7.6)$ & \\
\hline Telephone Visit, \%, (SD) & $12.7(25.4)$ & $12.8(26.2)$ & 0.8663 \\
\hline Clinical Video Telehealth, \% (SD) & $1.4(10.4)$ & $1.1(8.9)$ & 0.402 \\
\hline Home Telehealth, \%* & $477(25.5)$ & $440(19.6)$ & $<0.0001$ \\
\hline Nutrition/Weight Mgmt, \% & & & 0.4136 \\
\hline $0-1$ Visits & $1422(76)$ & $1721(76.6)$ & \\
\hline 2-5 Visits & $311(16.6)$ & $345(15.4)$ & \\
\hline $6+$ Visits & $138(7.4)$ & $182(8.1)$ & \\
\hline Pharmacy follow-up duration (SD)* & & & $<0.0001$ \\
\hline$<6$ Months & $342(18.3)$ & $636(28.3)$ & \\
\hline 6-12 Months & $517(27.6)$ & $579(25.8)$ & \\
\hline$\geq 12$ Months & $1012(54.1)$ & $1033(46)$ & \\
\hline Baseline A1c, \% (SD) & $9.9(1.4)$ & $10(1.8)$ & 0.0002 \\
\hline Creatinine Clearance, \%* & & & 0.0394 \\
\hline$\geq 60 \mathrm{ml} / \mathrm{min}$ & $1517(81.1)$ & $1869(83.1)$ & \\
\hline $30-59 \mathrm{ml} / \mathrm{min}$ & $214(11.4)$ & $259(11.5)$ & \\
\hline$<30 \mathrm{ml} / \mathrm{min}$ & $25(1.3)$ & $25(1.1)$ & \\
\hline Unknown & $115(6.2)$ & $95(4.2)$ & \\
\hline BP, baseline, $\mathrm{mmHg}$ (SD) & $134 / 77(18 / 12)$ & $134 / 77(17 / 12)$ & $0.267 / 0.3938$ \\
\hline$B P$, final, $\mathrm{mmHg}(\mathrm{SD})$ & $133 / 76(19 / 13)$ & $133 / 76(18 / 12)$ & $0.4081 / 0.2712$ \\
\hline \multicolumn{4}{|l|}{ Lipid levels, mg/dl, baseline: } \\
\hline Total Cholesterol (SD) & $160.7(46.1)$ & $174.1(55.8)$ & 0.1753 \\
\hline Triglycerides $(\mathrm{SD})^{\circ}$ & $197.7(173.7)$ & $242.5(264.9)$ & 0.001 \\
\hline $\mathrm{HDL}(\mathrm{SD})$ & $37(10)$ & $36.9(9.7)$ & 0.5502 \\
\hline $\operatorname{LDL}(S D)$ & $93.7(35.4)$ & $94.7(37.8)$ & 0.3881 \\
\hline \multicolumn{4}{|l|}{ Lipid levels, mg/dl, final visit } \\
\hline Total Cholesterol (SD)* & $160.7(46.1)$ & $154.4(44.4)$ & $<0.0001$ \\
\hline Triglycerides (SD) ${ }^{\circ}$ & $197.7(173.7)$ & $179.9(137.1)$ & 0.0006 \\
\hline $\mathrm{HDL}(\mathrm{SD})$ & $36.8(9.4)$ & $36.7(9.4)$ & 0.8796 \\
\hline $\operatorname{LDL}(S D)^{\circ}$ & $88.3(34.7)$ & $84.8(33.2)$ & 0.0016 \\
\hline Test Strips/day, mean no. (SD)* & $2.7(1.3)$ & $2.4(1.4)$ & $<0.0001$ \\
\hline
\end{tabular}




\begin{tabular}{|c|c|c|c|}
\hline & $\begin{array}{l}\text { Unsuccessful } \\
\text { Trajectory } \\
\text { (n= 1871) }\end{array}$ & $\begin{array}{l}\text { Successful } \\
\text { Trajectory } \\
\text { ( } \mathrm{n}=\mathbf{2 2 4 8})\end{array}$ & P-Value \\
\hline \multicolumn{4}{|l|}{ Medications, Baseline } \\
\hline Total, no. (SD) ${ }^{\diamond}$ & $2.12(0.97)$ & $1.94(1)$ & $<0.0001$ \\
\hline Metformin, \% & $1003(53.6)$ & $1241(55.2)$ & 0.3055 \\
\hline Sulfonylurea, \% & $681(36.4)$ & $823(36.6)$ & 0.8878 \\
\hline DPP-4 Inhibitor, \% & $138(7.4)$ & $182(8.1)$ & 0.3898 \\
\hline GLP-1 Agonist, \%* & $30(1.6)$ & $19(0.9)$ & 0.0254 \\
\hline SGLT-2 Inhibitor, \% & $2(0.1)$ & $1(0)$ & 0.594 \\
\hline Thiazolidinedione, \% & $52(2.8)$ & $70(3.1)$ & 0.5282 \\
\hline Insulin, \%* & & & $<0.0001$ \\
\hline Basal/Bolus & $805(43)$ & $773(34.4)$ & \\
\hline Basal Only & $390(20.8)$ & $449(20)$ & \\
\hline Prandial Only & $47(2.5)$ & $31(1.4)$ & \\
\hline U-500 & $5(0.3)$ & $4(0.2)$ & \\
\hline Statin, $\%$ & $1270(67.9)$ & $1491(66.3)$ & 0.2913 \\
\hline \multicolumn{4}{|l|}{ Medications, final visit } \\
\hline Total, no. $(S D)^{\diamond}$ & $2.92(0.95)$ & $2.65(0.97)$ & $<0.0001$ \\
\hline Metformin, \%* & $1216(65)$ & $1582(70.4)$ & 0.0002 \\
\hline Sulfonylurea, \%* & $571(30.5)$ & 759 (33.8) & 0.0266 \\
\hline DPP-4 Inhibitor, \% & 410 (21.9) & $474(21.1)$ & 0.5193 \\
\hline GLP-1 Agonist, \%* & $326(17.4)$ & $321(14.3)$ & 0.0058 \\
\hline SGLT-2 Inhibitor, \% & $118(6.3)$ & $122(5.4)$ & 0.2301 \\
\hline Thiazolidinedione, \% & $125(6.7)$ & $144(6.4)$ & 0.7219 \\
\hline Insulin, \%* & & & $<0.0001$ \\
\hline Basal/Bolus & $1116(59.7)$ & $975(43.4)$ & \\
\hline Basal Only & $428(22.9)$ & $554(24.6)$ & \\
\hline Prandial Only & $22(1.2)$ & $22(1)$ & \\
\hline U-500 & $8(0.4)$ & $9(0.4)$ & \\
\hline Statin, \% & $1540(82.3)$ & $1860(82.7)$ & 0.7166 \\
\hline \multicolumn{4}{|l|}{ Comorbid Conditions, \% } \\
\hline Alcohol Use Disorder & $148(7.9)$ & $203(9)$ & 0.1999 \\
\hline Substance Use Disorder & $365(19.5)$ & $471(21)$ & 0.2514 \\
\hline Hypertension* & $1702(91)$ & $1994(88.7)$ & 0.017 \\
\hline Heart Failure* & $245(13.1)$ & $235(10.5)$ & 0.0085 \\
\hline A1c at goal within second year* & $188(10.1)$ & $1168(54.6)$ & $<0.0001$ \\
\hline \multicolumn{4}{|c|}{$*: p<0.05$ (t-test), ${ }^{0}: p<0.05$ (Wilcoxon Rank Sum) } \\
\hline $\begin{array}{l}B M I=\text { Body mass index }, B P=B l o \\
L D L=L o w \text { density lipoproteins, } D \\
G L P-1=\text { Glucagon-lke peptide } 1\end{array}$ & $\begin{array}{l}\text { sure, } H D L=\text { High de } \\
\text { Dipeptidyl peptidase } \\
\text { = Sodium-glucose cc }\end{array}$ & $\begin{array}{l}\text { lipoproteins, } \\
\text { ssporter } 2\end{array}$ & \\
\hline
\end{tabular}


Those in groups classified as 'Successful' more often had a shorter duration of pharmacist follow-up and were less often enrolled in the home telehealth program. The home telehealth program is a program where Veterans can transmit health monitoring data (e.g. blood glucose readings) to registered nurses at the medical center to be provided to their diabetes provider. Demographics, modality of care delivery, nutrition clinic utilization, renal function, baseline $\mathrm{HbA1c}$, and measures of blood pressure and lipid control were similar between the two groups of trajectories. While statistically significant, there was not a clinically meaningful difference between 'Successful' and 'Unsuccessful' trajectories in measured co-morbidities. Additionally, those in 'Successful' trajectories were more often prescribed metformin and less often prescribed Glucagon-like peptide 1 (GLP-1) agonists and basal/bolus insulin regimens. As would be expected, a significantly greater proportion of patients in 'Successful' trajectory groups attained goal $\mathrm{HbAlc}$ within the second study year (defined as at least one $\mathrm{HbA} 1 \mathrm{c}$ value $\leq 7 \%$ between 12-24 months).

While those classified as following a successful trajectory group, on average, tended to show greater $\mathrm{HbA} 1 \mathrm{c}$ during most time intervals, as seen in Table 3, the main difference appears to be a much larger improvement during the first three months, possibly indicating this could be a critical time for setting the stage for success. Of note, if subjects had missing HbA1c measurements at either end of the time interval, they were not included in this sub-analysis. 
Table 3: Change in HbA1c percentage points per study time interval

\begin{tabular}{c|r|r|r|r|r|r}
\hline & \multicolumn{3}{|c|}{ Unsuccessful } & \multicolumn{3}{c}{ Successful } \\
Interval (Months) & Mean HbA1c Change (\%) & SD & n & Mean HbA1c Change (\%) & SD & $\mathbf{n}$ \\
\hline 0 to 3 & -0.91 & 1.45 & 1539 & -2.09 & 1.94 & 1945 \\
3 to 6 & -0.2 & 1.22 & 1211 & -0.38 & 1.04 & 1456 \\
6 to 9 & 0.02 & 1.25 & 976 & -0.15 & 0.98 & 1057 \\
9 to 12 & -0.08 & 1.33 & 876 & -0.23 & 1 & 879 \\
12 to 15 & -0.16 & 1.18 & 831 & -0.3 & 1.11 & 777 \\
15 to 18 & -0.18 & 1.29 & 784 & -0.29 & 1.02 & 737 \\
18 to 21 & -0.14 & 1.39 & 735 & -0.11 & 1.03 & 691 \\
21 to 24 & -0.23 & 1.21 & 706 & -0.2 & 0.98 & 586 \\
\hline
\end{tabular}




\subsection{Predictors of Successful Trajectories}

Logistic regression was completed to identify potential predictors of patients in successful trajectory groups versus unsuccessful, shown in Table 4.

Table 4: Predictors of Successful Trajectories

\begin{tabular}{|c|c|c|c|c|c|}
\hline \multirow{2}{*}{ Predictor } & & \multirow{2}{*}{ OR } & \multicolumn{2}{|c|}{ 95\% Confidence Limits } & \multirow{2}{*}{$p$} \\
\hline & & & Lower & Upper & \\
\hline Intercept & & - & - & - & 0.0532 \\
\hline Age & & 1.009 & 1.002 & 1.016 & 0.0101 \\
\hline \multicolumn{5}{|c|}{ Duration of Pharmacy Follow-up (Ref: < 6 Months) } & $<0.0001$ \\
\hline 6-12 Months & & 0.568 & 0.473 & 0.683 & $<0.0001$ \\
\hline$\geq 12$ Months & & 0.532 & 0.448 & 0.63 & $<0.0001$ \\
\hline Home Telehealth & & 0.796 & 0.681 & 0.93 & 0.0041 \\
\hline \multicolumn{5}{|c|}{ Nutrition Clinic Follow-up (Ref: 0-1 visits) } & 0.0853 \\
\hline $2-5$ visits & & 0.989 & 0.828 & 1.181 & 0.8995 \\
\hline $6+$ visits & & 1.307 & 1.027 & 1.667 & 0.0303 \\
\hline \multicolumn{5}{|c|}{ Creatinine Clearance, baseline (Ref: $\geq 60 \mathrm{ml} / \mathrm{min}$ ) } & 0.0091 \\
\hline $30-59 \mathrm{ml} / \mathrm{min}$ & & 0.884 & 0.715 & 1.094 & 0.2564 \\
\hline$<30 \mathrm{ml} / \mathrm{min}$ & & 0.733 & 0.409 & 1.312 & 0.2934 \\
\hline Unknown & & 0.626 & 0.468 & 0.834 & 0.0014 \\
\hline Test strips/day & & 0.936 & 0.884 & 0.991 & 0.0225 \\
\hline \multicolumn{5}{|c|}{ Insulin at Baseline (Ref: None) } & $<0.0001$ \\
\hline Basal/Bolus & & 0.701 & 0.594 & 0.826 & $<0.0001$ \\
\hline Basal only & & 0.815 & 0.681 & 0.976 & 0.0261 \\
\hline Prandial only & & 0.439 & 0.269 & 0.707 & 0.0008 \\
\hline$U-500$ & & 0.61 & 0.149 & 2.342 & 0.4672 \\
\hline \multicolumn{5}{|c|}{ Medical Center (Ref: 1) } & $<0.0001$ \\
\hline & 2 & 0.558 & 0.338 & 0.915 & 0.0215 \\
\hline & 3 & 0.889 & 0.565 & 1.389 & 0.6063 \\
\hline & 4 & 1.057 & 0.678 & 1.635 & 0.8051 \\
\hline & 5 & 0.592 & 0.362 & 0.959 & 0.0342 \\
\hline & 6 & 0.88 & 0.54 & 1.425 & 0.6043 \\
\hline & 7 & 1.027 & 0.664 & 1.577 & 0.9026 \\
\hline & 8 & 0.903 & 0.57 & 1.423 & 0.6624 \\
\hline
\end{tabular}

Hosmer and Lemeshow Goodness of Fit Test $(p=0.1389)$

Predicted vs. Observed Percent Concordant $=61.9 \%$ 
Age, gender, race, medical center, duration of CPS management, use of home telehealth, nutrition clinic utilization, level of renal function, number of test strips dispensed, total number of medication classes prescribed for diabetes at baseline, type of insulin prescribed at baseline, diagnosis of alcohol use disorder or substance use disorder during the study period, and the proportion of CPS visits conducted via telephone or clinical video telehealth were initially entered into the model as potential predictors (see Appendix $\mathrm{C}$ for the full model). In order to obtain greater parsimony, after backwards elimination with a significance level to stay in the model of 0.1 , the predictors in Table 4 above remained in the final model.

Older age, shorter duration of pharmacist management, no use of the home telehealth program, extent of follow-up with nutrition clinic services, lower average number of glucose test strips provided per day, not utilizing insulin at baseline were significant predictors of patients being classified into a successful trajectory group. While level of creatinine clearance appeared to be a significant predictor of success, though the meaning of this is unclear as this seems to have been driven by those classified as 'Unknown' due to missing data for one or more of baseline height, weight, or serum creatinine level. Additionally, the medical center where care was provided was significantly associated with success indicating potential practice or patient population differences across different VA institutions. 


\subsection{Relationship to Hospital Admissions}

Hospital admissions to non-psychiatric acute care units (e.g. excluding units such as psychiatric, skilled nursing, or domiciliary) were measured for each patient within the two-year study period. ${ }^{33}$ Logistic regression was performed to determine potential predictors of either all-cause or diabetes-related hospital admission. Initial models included predictors of age, gender, race, comorbidities, renal function, baseline $\mathrm{HbA1c}$, VA medical center, and assigned glycemic control trajectory group (for the full models, see Appendix C). In order to obtain greater parsimony, models were then evaluated via backwards elimination with a significance level to stay in the model of 0.1 . 
The predictors in Table 5 below remained in the final model for occurrence of all-cause hospital admission after backwards elimination of predictors.

Table 5: Predictors of all-cause hospital admission

\begin{tabular}{|c|c|c|c|c|}
\hline \multirow{2}{*}{ Predictor (Any Admission) } & \multirow{2}{*}{ OR } & \multicolumn{2}{|c|}{ 95\% Confidence Limits } & \multirow{2}{*}{$\mathrm{p}$} \\
\hline & & Lower & Upper & \\
\hline Intercept & $-\quad$ & - & - & $<0.0001$ \\
\hline Age & 1.016 & 1.008 & 1.025 & 0.0002 \\
\hline Heart Failure & 2.672 & 2.178 & 3.277 & $<0.0001$ \\
\hline Alcohol Use Disorder & 2.069 & 1.622 & 2.635 & $<0.0001$ \\
\hline Substance Use Disorder & 1.625 & 1.358 & 1.941 & $<0.0001$ \\
\hline \multicolumn{2}{|c|}{ Creatinine Clearance, baseline (Ref: $\geq 60 \mathrm{ml} / \mathrm{min}$ ) } & & & $<0.0001$ \\
\hline $30-59 \mathrm{ml} / \mathrm{min}$ & 1.469 & 1.169 & 1.842 & 0.0009 \\
\hline$<30 \mathrm{ml} / \mathrm{min}$ & 2.738 & 1.511 & 4.991 & 0.0009 \\
\hline Unknown & 0.757 & 0.519 & 1.081 & 0.1365 \\
\hline Baseline $\mathrm{HbA1c}$ & 1.112 & 1.043 & 1.186 & 0.0012 \\
\hline \multicolumn{2}{|c|}{ Trajectory Group (Ref: Moderate Early Decline) } & & & 0.0276 \\
\hline Large Early Decline & 0.645 & 0.418 & 0.989 & 0.0461 \\
\hline High Gradually Decreasing & 1.219 & 0.891 & 1.662 & 0.2129 \\
\hline Uncontrolled Stable & 0.984 & 0.83 & 1.116 & 0.8536 \\
\hline Early Decline/Subsequent Increase & 1.241 & 0.911 & 1.679 & 0.1663 \\
\hline Very Uncontrolled Stable & 1.131 & 0.72 & 1.755 & 0.5875 \\
\hline \multicolumn{2}{|l|}{ Medical Center (Ref: 1) } & & & 0.004 \\
\hline 2 & 0.786 & 0.444 & 1.422 & 0.4169 \\
\hline 3 & 1.071 & 0.646 & 1.832 & 0.7951 \\
\hline 4 & 1.172 & 0.716 & 1.981 & 0.5402 \\
\hline 5 & 1.123 & 0.658 & 1.969 & 0.6778 \\
\hline 6 & 1.196 & 0.7 & 2.101 & 0.5213 \\
\hline 7 & 0.821 & 0.506 & 1.379 & 0.439 \\
\hline 8 & 0.805 & 0.478 & 1.395 & 0.426 \\
\hline \multicolumn{4}{|c|}{ Hosmer and Lemeshow Goodness of Fit Test ( $p=0.6594)$} & \\
\hline Predicted vs. Observed Percent C & ordant $=6$ & $5.7 \%$ & & \\
\hline
\end{tabular}

As would be expected, more advanced age, diagnoses of heart failure, alcohol use disorder, or substance use disorder, greater renal dysfunction, and greater baseline HbAlc were all associated with a greater odds of all-cause hospital admission. However, once baseline HbA1c was accounted for, trajectory group membership did not seem to affect odds of all-cause admission in 
a meaningful way except possibly a slightly lower odds of hospitalization in the 'Large Early

Decline' group as compared to the 'Moderate Early Decline' group.

However, as seen in Table 6 below, assigned trajectory group did seem to be associated with differences in risk of diabetes-related admission.

Table 6: Predictors of diabetes-related hospital admission

\begin{tabular}{|c|c|c|c|c|}
\hline \multirow{2}{*}{ Predictor (DM Admission) } & \multirow{2}{*}{ OR } & \multicolumn{2}{|c|}{ 95\% Confidence Limits } & \multirow{2}{*}{$\mathrm{p}$} \\
\hline & & Lower & Upper & \\
\hline Intercept & - & - & - & $<0.0001$ \\
\hline Alcohol Use Disorder & 2.086 & 1.326 & 3.199 & 0.001 \\
\hline Substance Use Disorder & 1.921 & 1.341 & 2.724 & 0.0003 \\
\hline \multicolumn{2}{|c|}{ Creatinine Clearance, baseline (Ref: $\geq 60 \mathrm{ml} / \mathrm{min}$ ) } & & & 0.0005 \\
\hline $30-59 \mathrm{ml} / \mathrm{min}$ & 1.978 & 1.27 & 2.991 & 0.0017 \\
\hline$<30 \mathrm{ml} / \mathrm{min}$ & 4.421 & 1.487 & 10.611 & 0.0025 \\
\hline Unknown & 0.926 & 0.385 & 1.891 & 0.8486 \\
\hline \multicolumn{2}{|c|}{ Trajectory Group (Ref: Moderate Early Decline) } & & & $<0.0001$ \\
\hline Large Early Decline & 0.886 & 0.302 & 2.085 & 0.8014 \\
\hline High Gradually Decreasing & 3.314 & 1.914 & 5.618 & $<0.0001$ \\
\hline Uncontrolled Stable & 1.797 & 1.191 & 2.74 & 0.0056 \\
\hline Early Decline/Subsequent Increase & 3.628 & 2.049 & 6.261 & $<0.0001$ \\
\hline Very Uncontrolled Stable & 6.579 & 3.544 & 11.82 & $<0.0001$ \\
\hline \multicolumn{5}{|c|}{ Hosmer and Lemeshow Goodness of Fit Test $(p=0.7231)$} \\
\hline
\end{tabular}

Diagnosis of alcohol use disorder, substance use disorder, and worse baseline renal function remained important predictors of diabetes related hospital admissions. However, as opposed to solely baseline HbA1c level (see Appendix C for the full model), the assigned trajectory group of HbA1c change over time appeared to be related to diabetes-related admissions with the 'Large Early Decline' group showing no increased odds as compared to the 'Moderate Early Decline' group. Whereas the 'High Gradually Decreasing', 'Uncontrolled Stable', 'Early 
Decline/Subsequent Increase', and 'Very Uncontrolled Stable' groups all suggested higher odds of diabetes-related admission compared to the 'Moderate Early Decline' cohort.

Though these results regarding diabetes-related hospital admissions must be interpreted cautiously as they were exploratory in nature and as seen in Table 7 below, the absolute number of diabetes-related admissions was very small.

Table 7: Frequency of hospital admissions

\begin{tabular}{l|r|r}
\hline Glycemic Trajectory Group, $\mathbf{n}(\%)$ & Any Acute Admission & Any DM Admission \\
\hline High Gradually Decreasing & $106(32.6)$ & $23(7.1)$ \\
\hline Moderate Early Decline & $421(24.9)$ & $39(2.3)$ \\
Large Early Decline & $58(25.1)$ & $5(2.2)$ \\
Uncontrolled Stable & $369(25.1)$ & $58(4)$ \\
Early Decline/Subsequent Increase & $80(30.1)$ & $21(7.9)$ \\
Very Uncontrolled Stable & $44(32.1)$ & $18(13.1)$ \\
\hline
\end{tabular}


Chapter 4: Discussion 
Diabetes is an important public health problem and the use of clinical pharmacists in diabetes management has shown to improve attainment of goal HbA1c. ${ }^{1-2,21-26}$ In addition to the final HbA1c attained at a single time point, recent research has shown that the trajectory of how that HbA1c is attained over time is also likely important for outcomes such as mortality, microvascular complications, and even cognitive function. ${ }^{16-20}$ While likely helpful in the management of T2DM, clinical pharmacy specialists trained in diabetes management are likely a scarce resource as compared to the total number of patients with T2DM. Thus, it is important to either determine which subsets of patients are most likely to have clinical success with pharmacist management of diabetes and/or determine strategies to help patients move from less successful trajectories of glycemic control to more successful ones. Utilizing a large sample of over 4,000 Veterans from eight VA medical centers, this study provides the first known evaluation of the distinct trajectories of HbA1c change after initiation of CPS management of T2DM.

In this study, patients were able to be successfully segmented into six distinct trajectories of change in $\mathrm{HbA1c}$ over time after initiation of CPS management of diabetes (Figure 2 and Figure 3). These trajectories can be distinguished by the general range of starting HbAlc (e.g. moderately uncontrolled versus very uncontrolled), how glycemic control changes over time (e.g. increasing, unchanged, or decreasing), and how quickly HbA1c changes over time (e.g. early or gradual). A large majority of patients either fell into the 'Moderate Early Decline' group (41.1\%) or the 'Uncontrolled Stable' group (35.6\%). Interestingly, these glycemic control patterns diverge until around six months and then subsequently the trajectory lines in both groups 
plateau and are reasonably parallel. While a much smaller portion of the sample, a similar pattern is seen in the 'Large Early Decline' (5.6\%) group with a large decrease in HbAlc over the first six months with a subsequent plateau. Overall, on average, except for the 'High Gradually Decreasing' group (7.9\%), the first six months of CPS management appear crucial towards success at reaching therapeutic goals. This is further supported by the mean change in HbA1c over each 3-month time interval seen in Table 3, with the largest difference between successful and unsuccessful trajectories being in the 0 to 3-month timeframe. These results align with the recent VA study by Ourth et al. which showed that on average for their study population the greatest $\mathrm{HbA1c}$ improvement occurred within the first three months of either pharmacist management or usual care prior to plateauing. ${ }^{26}$ As CPS resources are relatively scarce as compared to the total number of patients suffering from diabetes, these data suggest possibly targeting those resources aggressively towards the first three to six months of management to have maximal impact. These data also suggest that it may be beneficial to examine pharmacist panels to identify and re-evaluate patients that are no longer achieving clinical progress with CPS management, focusing resources, to accept new patients with very uncontrolled $\mathrm{HbA1c}$. The reasons for this early initial progress and subsequent plateauing are uncertain. It may indicate possible importance to creating an effective start to the CPS/patient relationship.

Additionally, if the availability of clinical pharmacy specialist support is limited, it may be wise to prioritize patients with the most extreme baseline $\mathrm{HbAlc}$ readings (e.g. $\geq 11 \%$ ) first. Out of the three $\mathrm{HbA1c}$ trajectories with a baseline $\mathrm{HbA1c}$ in this range, approximately $80 \%$ of patients were classified into a trajectory group associated with a sustained reduction in HbA1c. 
Additionally, as most patients tended to fall into the 'Moderate Early Decline' or 'Uncontrolled Stable" groups with similar baseline HbA1c values, a next step for further investigation would be to identify interventions that can help patients achieve a glycemic control trajectory more similar to the 'Moderate Early Decline' group rather than the 'Uncontrolled Stable' group.

Another difference between those counted as being part of successful trajectories versus unsuccessful is that those following successful trajectories, on average, tended to have a shorter total duration of pharmacy follow-up. This could be related to achieving more reduction in $\mathrm{HbA1c}$ in the early months of CPS management. Also, though mean baseline HbA1c values were clinically similar, those patients classified as following unsuccessful trajectories were prescribed basal/bolus insulin. Additionally, being enrolled in the home telehealth program and being prescribed a greater number of glucose test strips were also associated with less likelihood of following a successful trajectory. These could indicate something about these patients that could be making them more difficult to manage. While not measured in this study, it could possibly be related to things such as duration of diabetes. Conversely, having had a total of more than 6 visits with the VA's nutrition or weight management clinics was associated with likelihood of following a successful trajectory. This could be possibly related to direct effects of working with VA dieticians or it could also be a marker for an overall greater patient commitment to improving their diabetes control. Finally, there appeared to be some difference in likelihood of following a successful trajectory based on the medical center where care was provided, potentially indicating some difference in results across different VA sites. 
Lastly, there did not seem to be a substantial effect of glycemic control trajectory on all-cause hospitalization occurrence once baseline HbA1c was accounted for. However, as may be expected, there possibly could be an association between trajectory of glycemic control and lower odds of diabetes-related hospital admission with patients assigned to trajectory groups where a greater proportion of patients reached HbA1c targets ('Large Early Decline' and 'Moderate Early Decline'). However, as noted, these results should be interpreted with caution given the exploratory nature of this analysis and the low absolute numbers of diabetes-related hospital admissions.

\section{Limitations}

As this was a retrospective analysis, not all patients had $\mathrm{HbA1c}$ measurements for each study time point, the number of HbA1c values available analysis at each 3-month interval is shown in Table 8.

Table 8: Available HbA1c Values at Each Time Interval

\begin{tabular}{c|c}
\hline Month & HbA1c Values (n) \\
\hline 0 & 4119 \\
\hline 3 & 3484 \\
\hline 6 & 3083 \\
\hline 9 & 2633 \\
\hline 12 & 2655 \\
\hline 15 & 2430 \\
\hline 18 & 2443 \\
\hline 21 & 2224 \\
\hline 24 & 2298 \\
\hline
\end{tabular}


While group-based trajectory modeling can accommodate data missing at random, it cannot be ruled out that there are commonalities between patients who did not have an HbA1c measured every three months, such as practice patterns of individual providers. ${ }^{31}$ Also, the trajectory groups that were determined are best fit measurements to a clustering of individual trajectories. Thus, the average trajectory pattern does not mean that individual patients follow that trajectory exactly. Some patients were not completely naïve to pharmacist management of diabetes. However, a one-year 'wash-out' period between any prior pharmacist management hopefully ensured capturing new episodes of pharmacist diabetes care. Also, there are several limitations associated with the use of retrospective data. Firstly, HbAlc measurements were completed as part of routine clinical care, and thus do not always occur exactly at the study interval breakpoints. While the most appropriate HbA1c value for each study interval was selected, patients may have had more than nine HbA1c measurements in the two-year study period and these 'extra' measurements are not taken into consideration in this analysis. However, given mechanistically how HbAlc is determined, we would not expect additional readings measured in the same time-frame to differ substantially in most cases. Additionally, the presence of comorbidities is reliant on proper coding of medical record problem lists, clinic visits, and admissions. Also, medication dispensing records do not necessarily mean the patient was actively using that medication at home. These data also do not provide an assessment of how trajectories of glycemic control with pharmacist management compares with trajectories achieved by usual care by solely primary care provider management. These data cannot be applied to patient cohorts with baseline HbAlc values $<8 \%$, these patients were excluded from the study cohort as many patients may have individualized $\mathrm{HbA} 1 \mathrm{c}$ targets between 7-8\% and patients with baseline HbA1c values in this range could be being managed by pharmacy 
providers for other reasons such as prevention of hypoglycemia or for other conditions such as hypertension where diabetes is assessed as a comorbidity rather than the primary focus of pharmacist management. Finally, these data are aggregated, so they do not give any insight into how practice patterns of any individual pharmacist provider results in a specific set of trajectories.

Also, overall, patient characteristics seemed similar for those classified into successful trajectory groups versus unsuccessful groups. It is likely that there are other unmeasured factors that contribute to a patient's trajectory of glycemic control, many of which are not able to be measured in a retrospective analysis. Some of these factors could include the quality of the patient/pharmacist relationship, the degree of trust between the patient and CPS, characteristics of social support networks, lifestyle behaviors (e.g. dietary choices and changes in physical activity), and socioeconomic factors. As expected, those classified into following successful trajectories were more likely to have achieved reaching an HbA1c goal of $\leq 7 \%$. However, with current recommendations to individualize treatment targets in some patients such as for those with a high degree of comorbidities or diabetes complications, more patients might have actually achieved their individualized HbA1c targets. ${ }^{13}$

Finally, while this study was able to show that there are subgroups of patients who have more success with pharmacy management of diabetes that are more likely to achieve goal HbAlc and that certain glycemic trajectories could confer greater risk of diabetes-related hospital admission, due to the relatively short study period length, this study is not able to provide evidence on the 
effect of these distinct trajectories on ultimate outcomes such as mortality or progression of diabetes complications.

\section{Considerations for Future Research}

It is an interesting finding that the first three to six months of pharmacist management may be crucial to determining a patient's longer-term success with this clinical service. Further study could elucidate reasons for this trend and potentially identify best practices that help more patients follow successful trajectories. There is also opportunity for conducting prospective investigations aimed at designing strategies to help patients move from less successful to more successful glycemic control trajectories. Also, it could be valuable to investigate how trajectories of diabetes control change after completion or cessation of pharmacist management of diabetes to determine if glycemic control tends to be maintained or if it degrades. It could also be compared how these trajectories of diabetes control with pharmacist diabetes management differ from trajectories of diabetes control of propensity matched controls managed solely by primary care providers. Additionally, considering the future of health informatics, there could someday be the possibility of utilizing data glycemic control trajectories in conjunction with real-time data from continuous glucose monitoring, potentially on wearable technology, from large populations to inform machine learning algorithms to power clinical decision support tools to inform patients and providers of a patient's current and anticipated trajectory helping clinicians and patients work together to improve that long-term outlook. Further study could be conducted on the difference in odds of diabetes-related hospital admission. Finally, it remains to be evaluated if the achievement of different trajectories of glycemic control through pharmacist management of 
diabetes results in differences in long-term ultimate outcomes including mortality, longer-term rates of hospitalization, quality of life, or rates of macrovascular or microvascular complications. 


\section{References}

1. Liu Y, Sayam S, Shao X, et al. Prevalence of and Trends in Diabetes Among Veterans, United States, 2005-2014. Prev Chronic Dis. 2017;14: E135.

2. Carls G, Huynh J, Tuttle E, et al. Achievement of glycated hemoglobin goals in the US remains unchanged through 2014. Diabetes Ther. 2017; 8(4): 863-873.

3. https://www.ncqa.org/hedis/measures/comprehensive-diabetes-care/. Accessed: October $18,2019$.

4. https://www.va.gov/QUALITYOFCARE/measure-up/SAIL_definitions.asp. Accessed: October 18, 2019.

5. The Diabetes Control and Complications Trial Research Group. The effect of intensive treatment of diabetes on the development and progression of long-term complications in insulin-dependent diabetes mellitus. N Engl J Med. 1993; 329(14): 977-986.

6. The DCCT/EDIC Research Group. Sustained effect of intensive treatment of type 1 diabetes mellitus on development and progression of diabetic nephropathy. JAMA. 2003;290: 2159-2167.

7. Martin CL, Albers JW, Pop-Busui R. Neuropathy and related findings in the diabetes control and complications trial/epidemiology of diabetes interventions and complications study. Diabetes Care. 2014; 37: 31-38.

8. UK Prospective Diabetes Study Group. Intensive blood-glucose control with sulfonylureas or insulin compared with conventional treatment and risk of complications in patients with type 2 diabetes (UKPDS 33). Lancet. 1998; 352: 837-853.

9. UK Prospective Diabetes Study Group. Effect of intensive blood-glucose control with metformin on complications in overweight patients with type 2 diabetes (UKPDS 34). Lancet. 1998; 352: 854-865.

10. The ADVANCE Collaborative Group. Intensive blood glucose control and vascular outcomes in patients with type 2 diabetes. $N$ Engl J Med. 2008; 358: 2560-2572.

11. The Action to Control Cardiovascular Risk in Diabetes Study Group. Effects of intensive glucose lowering in type 2 diabetes. N Engl J Med. 2008; 358: 2545-2559.

12. Duckworth WD, Abraira C, Moritz T, et al. Glucose control and vascular complications in veterans with type 2 diabetes. N Engl J Med. 2009; 360: 129-139.

13. American Diabetes Association. Standards of medical care in diabetes - 2019. Diabetes Care. 2019; 42(Suppl 1): S61-S70.

14. Helgeson VS, Vaughn AK, Seltman H, et al. Featured article: trajectories of glycemic control over adolescence and emerging adulthood: An 11-year longitudinal study of youth with type 1 diabetes. J Pediatr Psychol. 2018; 43(1): 8-18.

15. Walraven I, Mast MR, Hoekstra T, et al. Distinct HbA1c trajectories in a type 2 diabetes cohort. Acta Diabetol. 2015; 52: 267-275.

16. Laiteerapong N, Karter AJ, Moffet HH, et al. Ten-year hemoglobin A1c trajectories and outcomes in type 2 diabetes mellitus: the diabetes \& aging study. J Diabetes Complications. 2017; 31(1): 94-100.

17. Gebregziabher M, Egede LE, Lynch CP, et al. Effect of trajectories of glycemic control on mortality in type 2 diabetes: a semiparametric joint modeling approach. Am J Epidemiol. 2010; 171: 1090-1098.

18. Low S, Zhang X, Wang J, et al. Impact of haemoglobin A1c trajectories on chronic kidney disease progression in type 2 diabetes. Nephrology. 2019; 24: 1026-1032. 
19. Luo M, Lim WY, Tan CS, et al. Longitudinal trends in HbA1c and associations with comorbidity and all-cause mortality in Asian patients with type 2 diabetes: a cohort study. Diabetes Res Clin Pract. 2017; 133: 69-77.

20. Ravona-Springer R, Heymann A, Schmeidler J, et al. Trajectories of glycemic control over time are associated with cognitive performance in elderly subjects with type 2 diabetes. PLoS One. 2014; 9(6): e97384.

21. Greer N, Bolduc J, Geurkink E, Koeller E, Rector T, Olson K, MacDonald R, and Wilt TJ. Pharmacist-led Chronic Disease Management: A Systematic Review of Effectiveness and Harms Compared to Usual Care. VA ESP Project \#09-009; 2015.

22. Wassell K, Sullivan J, Jett BP, et al. Comparison of clinical pharmacy specialists and primary care physicians in treatment of type 2 diabetes mellitus in rural veterans affairs facilities. Am J Health-Syst Pharm. 2018; 75(suppl 1): S6-S12.

23. Schmidt K, Caudill J, Hamilton T. Impact of clinical pharmacy specialists on glycemic control in veterans with type 2 diabetes. Am J Health-Syst Pharm. 2019; 76(suppl 1): S9S14.

24. Schultz JL, Horner KE, McDanel DL, et al. Comparing clinical outcomes of a pharmacist-managed diabetes clinic to usual physician-based care. J Pharm Prac. 2018; 31(3): 268-271.

25. Maeng DD, Graham J, Bogart M, et al. Impact of a pharmacist-led diabetes management on outcomes, utilization, and cost. Clinicoecon Outcomes res. 2018; 10: 551-562.

26. Ourth HL, Hur K, Morreale AP, et al. Comparison of clinical pharmacy specialists and usual care in outpatient management of hyperglycemia in Veterans Affairs medical centers. Am H Health Syst Pharm. 2019; 76(1): 26-33.

27. Lam SW, Russo-Alvarez G, Cristiani C, et al. Development of an ambulatory clinical pharmacy prioritization prediction model for patients with diabetes. Annals of Pharmacotherapy. 2017; 51(1): 33-38.

28. US Department of Veterans Affairs. 172VA10P2: VHA Corporate Data Warehouse-VA. 79 FR 4377. Updated August 20, 2019. Last accessed September 28, 2019.

29. Jones BL, Nagin DS. Advances in group-based trajectory modeling and an SAS procedure for estimating them. Soc Methods \& Res. 2007; 35(4): 542-571.

30. Nagin DS, Odgers CL. Group-based trajectory modeling in clinical research. Ann Rev Clin Psychol. 2010; 6: 109-138.

31. Nagin DS. Group-Based Modelling of Development. Cambridge, MA: Harvard University Press, 2005.

32. Jones BL, Nagin DS, Roeder K. A SAS procedure based on mixture models for estimating developmental trajectories. Sociol Methods Res. 2001; 29(3): 374-393.

33. Vincent BM, Wiitala WL, Burns JA, et al. Using Veterans Affairs corporate data warehouse to identify 30-day hospital readmissions. Health Serv Outcomes Res Method. 2018; 18: 143-154. 


\section{Appendix A: Model Selection Procedure}

Table 8 illustrates the steps taken in order when developing the model used for determining the best fitting glycemic control trajectories for use in PROC TRAJ in SAS. First, the number of groups were increased successively by one with each additional group being added modeled with a quadratic equation except for one group modeled as a zero-order group as it was thought there would likely be a subset of patients with no significant HbAlc change. ${ }^{31}$

Table 9: Model selection procedure

\begin{tabular}{|c|c|c|c|c|l}
\hline Step & \# Groups & Group Orders & BIC & $\mathbf{2 *} \Delta$ BIC & \multicolumn{1}{|c}{ Comment } \\
\hline 1 & 2 & 0,2 & -46583.96 & - & \\
\hline 2 & 3 & $0,2,2$ & -45015.19 & 3137.54 & \\
\hline 3 & 4 & $0,2,2,2$ & -43963.37 & 2103.64 & \\
\hline 4 & 5 & $0,2,2,2,2$ & -43701.21 & 524.32 & \\
\hline 5 & 6 & $0,2,2,2,2,2$ & -43434.13 & 534.16 & Maximum 6 groups reached \\
\hline 6 & 6 & $0,3,2,2,2,2$ & -43036.65 & 794.96 & \\
\hline 7 & 6 & $0,4,2,2,2,2$ & -42943.86 & 185.58 & \\
\hline 8 & 6 & $0,5,2,2,2,2$ & -42977.56 & -67.4 & Return to order =4 for group 2 \\
\hline 9 & 6 & $0,4,3,2,2,2$ & -42597.99 & 691.74 & Step 7 = Null model \\
\hline 10 & 6 & $0,4,4,2,2,2$ & -42550.46 & 95.06 & \\
\hline 11 & 6 & $0,4,5,2,2,2$ & -42516.29 & 68.34 & \\
\hline 12 & 6 & $0,4,5,3,2,2$ & -42334.62 & 363.34 & \\
\hline 13 & 6 & $0,4,5,4,2,2$ & -42388.68 & -108.12 & Return to order =3 for group 4 \\
\hline 14 & 6 & $0,4,5,3,3,2$ & -42512.69 & -356.14 & Group 1 drops out of model \\
\hline 15 & 6 & $2,4,5,3,3,2$ & -42229 & 211.24 & Trial order = 2 for group 1; Step 12= Null model \\
\hline 16 & 6 & $2,4,5,3,4,2$ & -42406.34 & -354.68 & Return to order = 3 for group 5 \\
\hline 17 & 6 & $2,4,5,3,3,3$ & -42455.62 & -453.24 & Return to order = 2 for group 6 \\
\hline 18 & 6 & $2,4,5,3,3,2$ & -42229 & - & Final Model \\
\hline
\end{tabular}


The following table provides the parameter estimates defining the modeled trajectories of each group along with estimated proportions of group membership:

Table 10: Trajectory group parameter estimates

\begin{tabular}{|c|c|c|c|c|c|}
\hline \multicolumn{6}{|c|}{ Maximum Liklihood Estimates } \\
\hline \multicolumn{6}{|c|}{ Model: Censored Normal (cnorm) } \\
\hline Group & Parameter & Estimate & $\begin{array}{l}\text { Standard } \\
\text { Error }\end{array}$ & $\begin{array}{c}\text { T for } \mathrm{HO}: \\
\text { Parameter }=0\end{array}$ & Prob $>|\mathrm{T}|$ \\
\hline \multirow[t]{4}{*}{1} & Intercept & 11.38067 & 0.11751 & 96.85 & $<0.0001$ \\
\hline & Linear & -0.15149 & 0.01834 & -8.262 & $<0.0001$ \\
\hline & Quadratic & 0.00214 & 0.00066 & 3.236 & 0.0012 \\
\hline & Group Membership (\%) & 7.90151 & 0.81855 & 9.653 & $<0.0001$ \\
\hline \multirow[t]{6}{*}{2} & Intercept & 9.22345 & 0.03166 & 291.324 & $<0.0001$ \\
\hline & Linear & -0.74999 & 0.02357 & -31.816 & $<0.0001$ \\
\hline & Quadratic & 0.09634 & 0.0043 & 22.416 & $<0.0001$ \\
\hline & Cubic & -0.00497 & 0.00028 & -17.829 & $<0.0001$ \\
\hline & Quartic & 0.00009 & 0.00001 & 15.19 & $<0.0001$ \\
\hline & Group Membership (\%) & 39.98532 & 1.48293 & 26.964 & $<0.0001$ \\
\hline \multirow[t]{7}{*}{3} & Intercept & 13.41118 & 0.10319 & 129.963 & $<0.0001$ \\
\hline & Linear & 3.20694 & 0.10409 & -30.811 & $<0.0001$ \\
\hline & Quadratic & 0.60532 & 0.03122 & 19.392 & $<0.0001$ \\
\hline & Cubic & -0.05159 & 0.00359 & -14.381 & $<0.0001$ \\
\hline & Quartic & 0.00202 & 0.00017 & 11.726 & $<0.0001$ \\
\hline & Quintic & -0.00003 & 0 & -10.108 & $<0.0001$ \\
\hline & Group Membership (\%) & 5.73527 & 0.45764 & 12.532 & $<0.0001$ \\
\hline \multirow[t]{5}{*}{4} & Intercept & 9.41683 & 0.03957 & 237.982 & $<0.0001$ \\
\hline & Linear & -0.25646 & 0.0133 & -19.282 & $<0.0001$ \\
\hline & Quadratic & 0.02028 & 0.00137 & 14.808 & $<0.0001$ \\
\hline & Cubic & -0.00048 & 0.00004 & -12.628 & $<0.0001$ \\
\hline & Group Membership (\%) & 35.87022 & 1.3047 & 27.493 & $<0.0001$ \\
\hline \multirow[t]{5}{*}{5} & Intercept & 10.28422 & 0.08753 & 117.489 & $<0.0001$ \\
\hline & Linear & -0.4484 & 0.0458 & -9.79 & $<0.0001$ \\
\hline & Quadratic & 0.05171 & 0.00499 & 10.372 & $<0.0001$ \\
\hline & Cubic & -0.00135 & 0.00013 & -10.241 & $<0.0001$ \\
\hline & Group Membership (\%) & 7.16628 & 0.71797 & 9.981 & $<0.0001$ \\
\hline \multirow[t]{4}{*}{6} & Intercept & 12.15828 & 0.09593 & 126.747 & $<0.0001$ \\
\hline & Linear & -0.01282 & 0.02011 & -0.637 & 0.5238 \\
\hline & Quadratic & 0.00091 & 0.00089 & 1.026 & 0.3051 \\
\hline & Group Membership (\%) & 3.34139 & 0.31502 & 10.607 & $<0.0001$ \\
\hline$\sigma$ & & 1.07433 & 0.00516 & 208.153 & $<0.0001$ \\
\hline
\end{tabular}




\section{Appendix B: Trajectory Model Diagnostics}

Based on Nagin (2005), three assessments of fit of the data to the modeled glycemic control trajectory groups were assessed. ${ }^{33}$ These included an assessment of the mean posterior probabilities of assignment to each group where mean posterior probabilities $>0.7$ indicate acceptable fit to the data, the odds of correct classification to the assigned trajectory group with values $>5$ indicating acceptable fit to the data, and ratio of estimated group probabilities to the proportion of the sample assigned to each group which should be near one for each group. ${ }^{33}$

\section{Mean Posterior Group Probabilities}

Table 11: Mean posterior group probabilities

\begin{tabular}{|c|c|c|c|c|c|c|}
\hline & \multicolumn{6}{|c|}{ Mean Group Posterior Probability } \\
\hline & 1 & 2 & 3 & 4 & 5 & 6 \\
\hline \multirow{6}{*}{$\begin{array}{l}\frac{0}{3} \\
\frac{0}{0}\end{array}$} & 0.80615 & 0.00175 & 0.01503 & 0.07946 & 0.07852 & 0.01808 \\
\hline & 0.00018 & 0.86679 & 0.01221 & 0.11962 & 0.00118 & 0.00001 \\
\hline & 0.01503 & 0.05856 & 0.86654 & 0.04893 & 0.00929 & 0.00166 \\
\hline & 0.02452 & 0.11316 & 0.00636 & 0.83061 & 0.0252 & 0.00015 \\
\hline & 0.071 & 0.00065 & 0.00437 & 0.06997 & 0.83901 & 0.015 \\
\hline & 0.0351 & 0.000003 & 0.0001 & 0.00019 & 0.03657 & 0.92803 \\
\hline
\end{tabular}




\section{Odds of Correct Classification}

"Odds of Correct Classification $=\frac{\frac{\text { Mean Posterior Probability }}{1-\text { Mean Posterior Probability }}}{\frac{\text { Estimated Population Rate }}{1-\text { Estimated Population Rate }}}, 33$

Table 12: Odds of correct classification

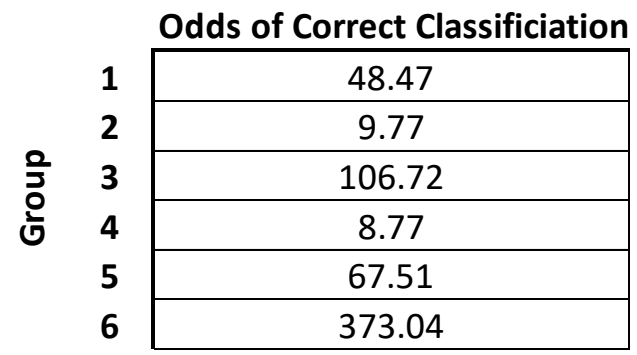

\section{Ratio of Estimated Group Probabilities to the Proportion Assigned to Each Group}

Table 13: Ratio of estimated group probabilities to proportion assigned to each group

\begin{tabular}{|l|l|l|}
\cline { 2 - 3 } & $\mathbf{1}$ & 1.0014 \\
\cline { 3 - 3 } & $\mathbf{2}$ & 0.9734 \\
\cline { 3 - 4 } & $\mathbf{3}$ & 1.0227 \\
\hline \multirow{2}{*}{$\mathbf{0}$} & $\mathbf{4}$ & 1.0065 \\
\cline { 3 - 4 } & $\mathbf{5}$ & 1.1097 \\
\cline { 3 - 3 } & $\mathbf{6}$ & 1.0046 \\
\hline
\end{tabular}

\section{Reasonableness Check for Glycemic Trajectory Group Assignments}

A random sample of 50 patients was drawn from each trajectory group via PROC

SURVEYSELECT in SAS using simple random sampling. Those patient's HbA1c values versus time were plotted to aid in confidence that the average trajectory paths that were determined are reasonable estimations of actual patient trajectories in the sample. Results of this random sampling for each trajectory group are displayed below in Figures 4 through 9. 


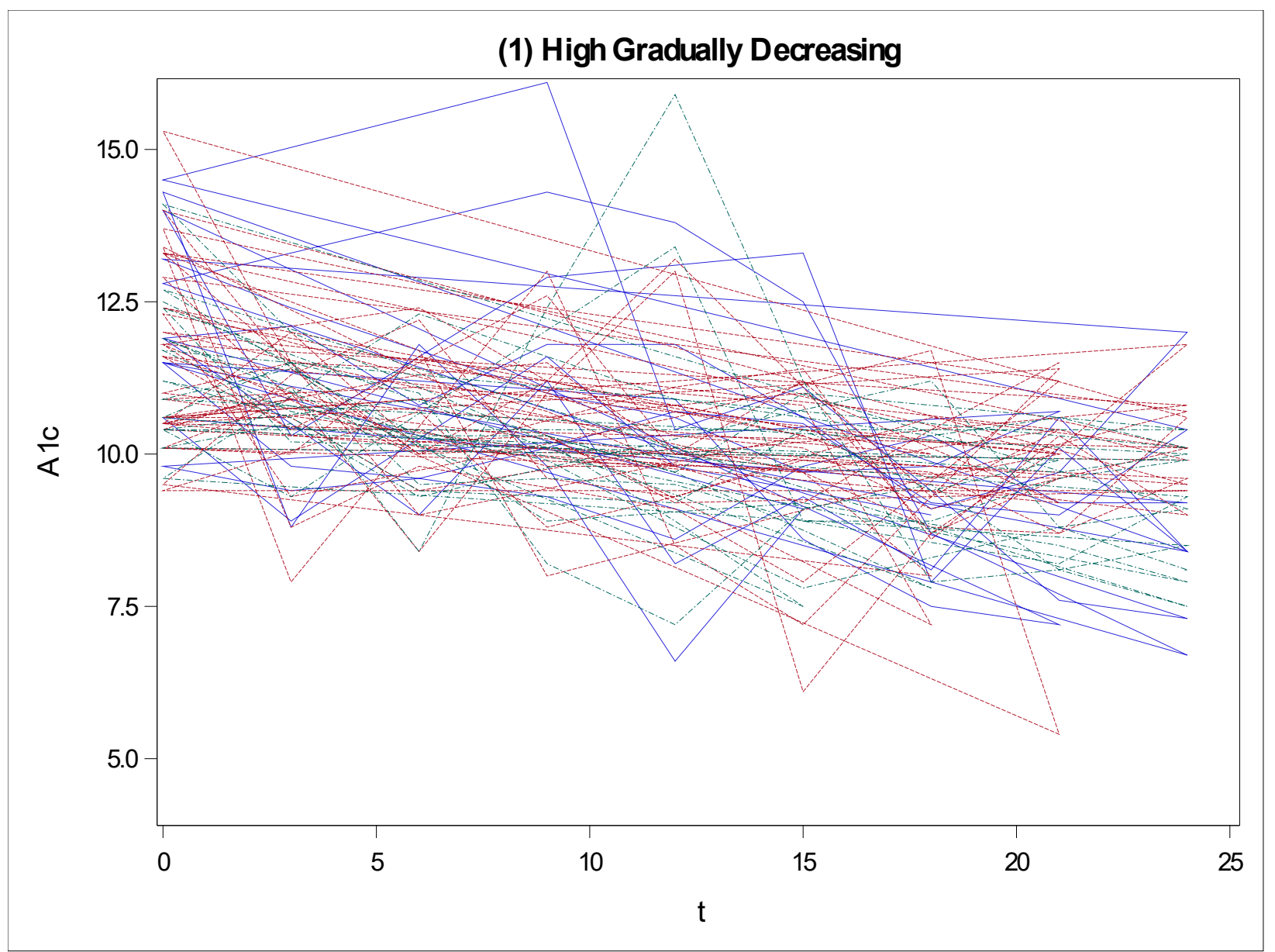

Figure 4: High Gradually Decreasing Example Trajectories 


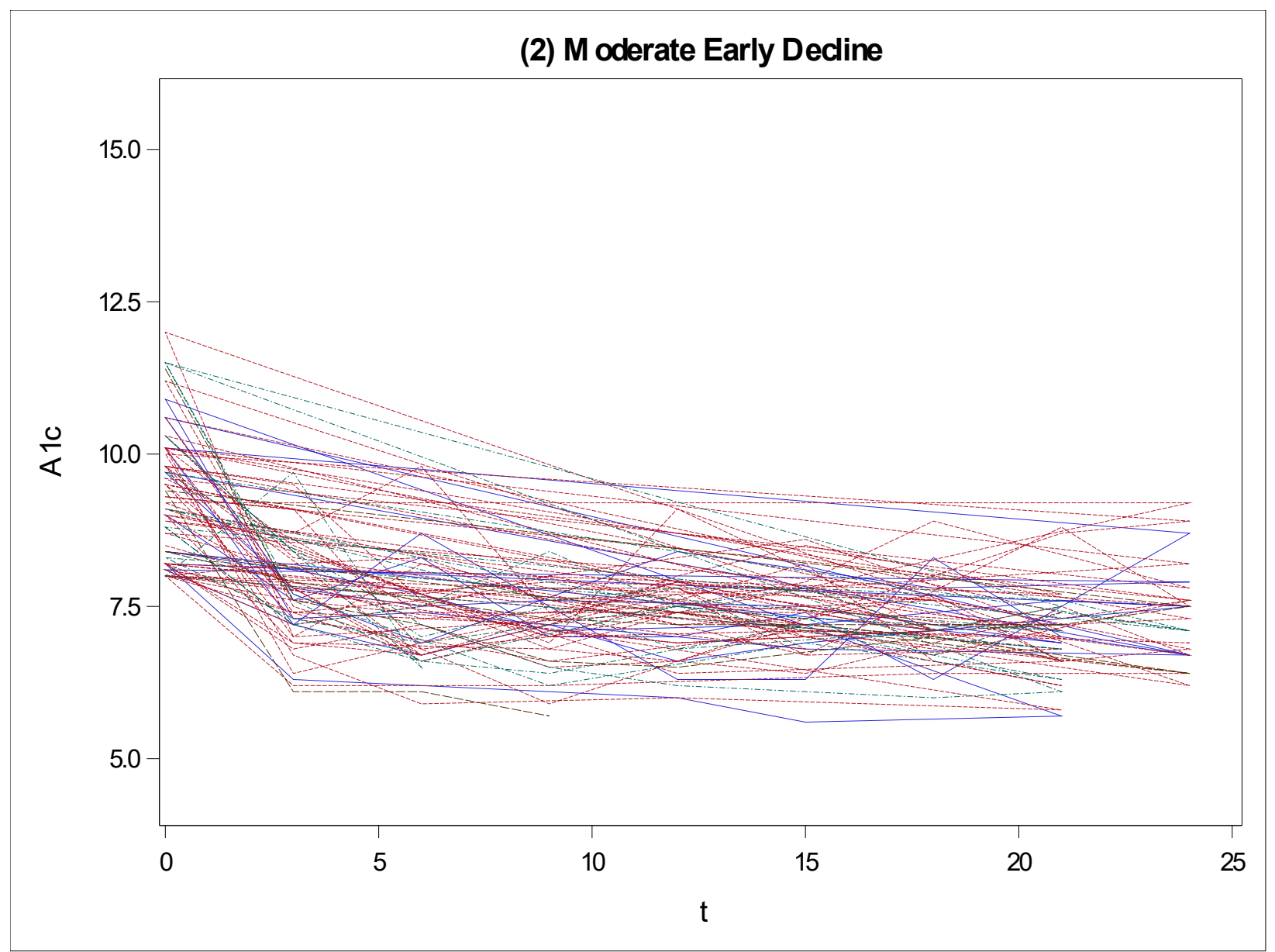

Figure 5: Moderate Early Decline Example Trajectories 


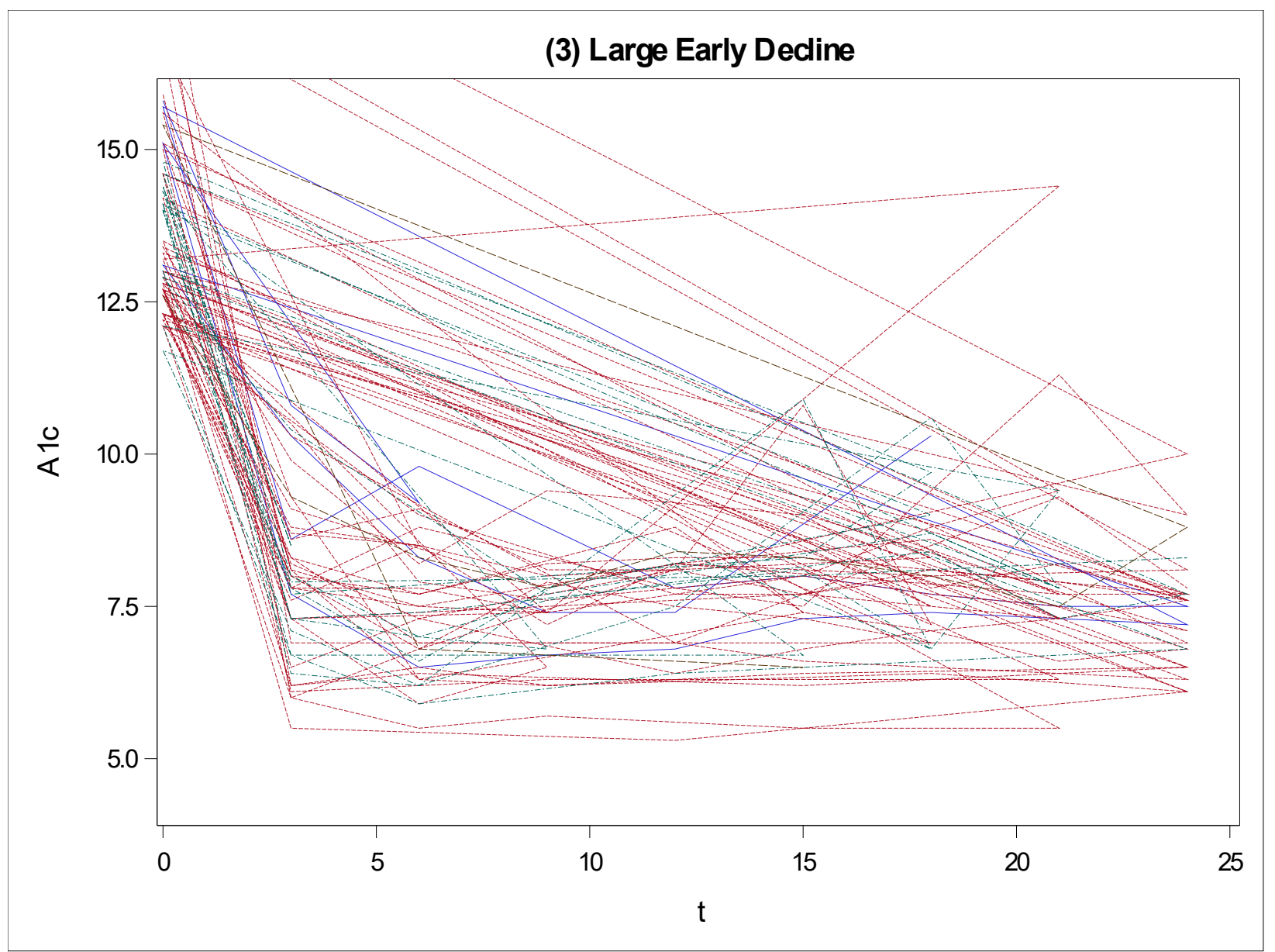

Figure 6: Large Early Decline Example Trajectories 


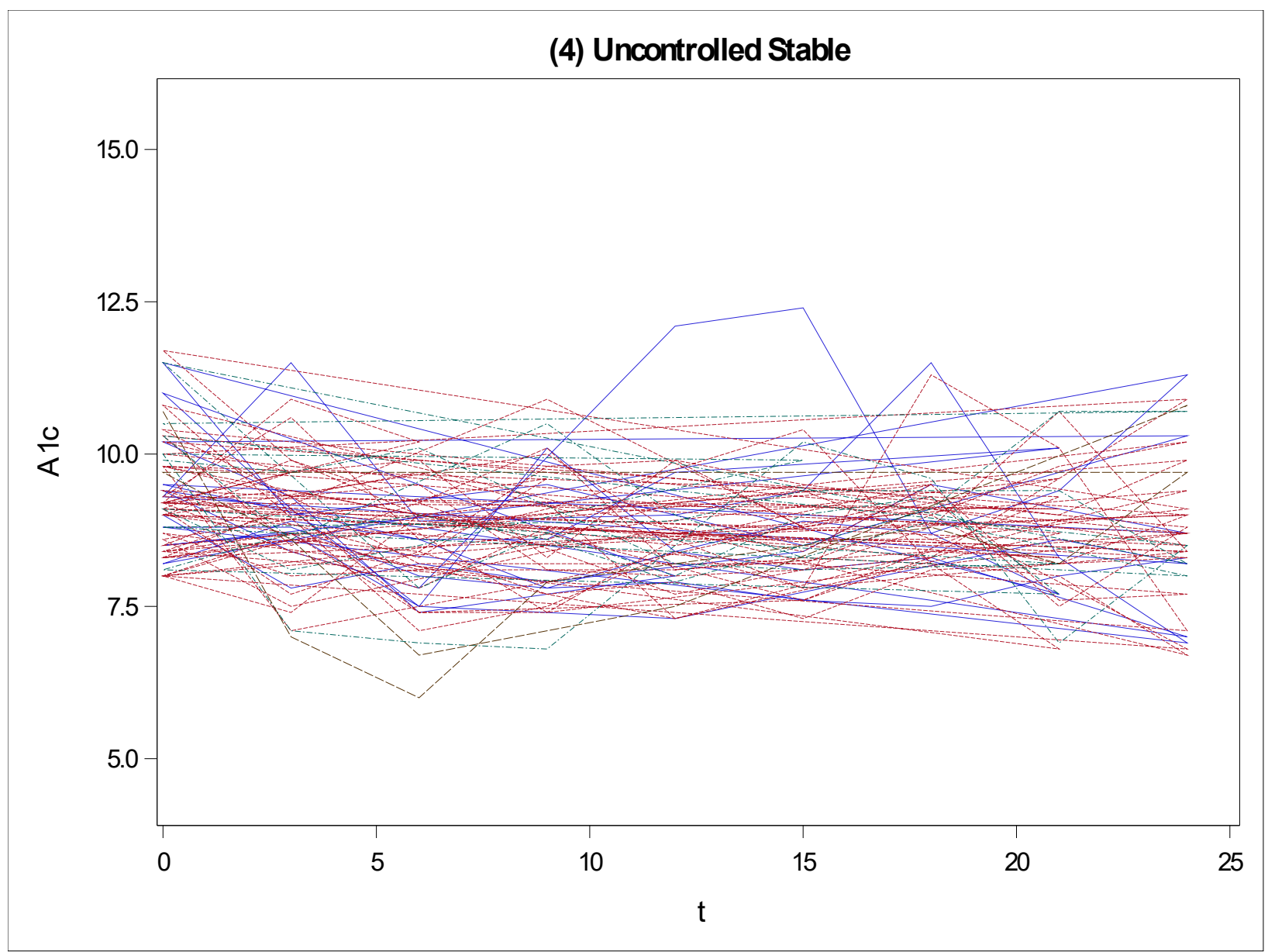

Figure 7: Uncontrolled Stable Example Trajectories 


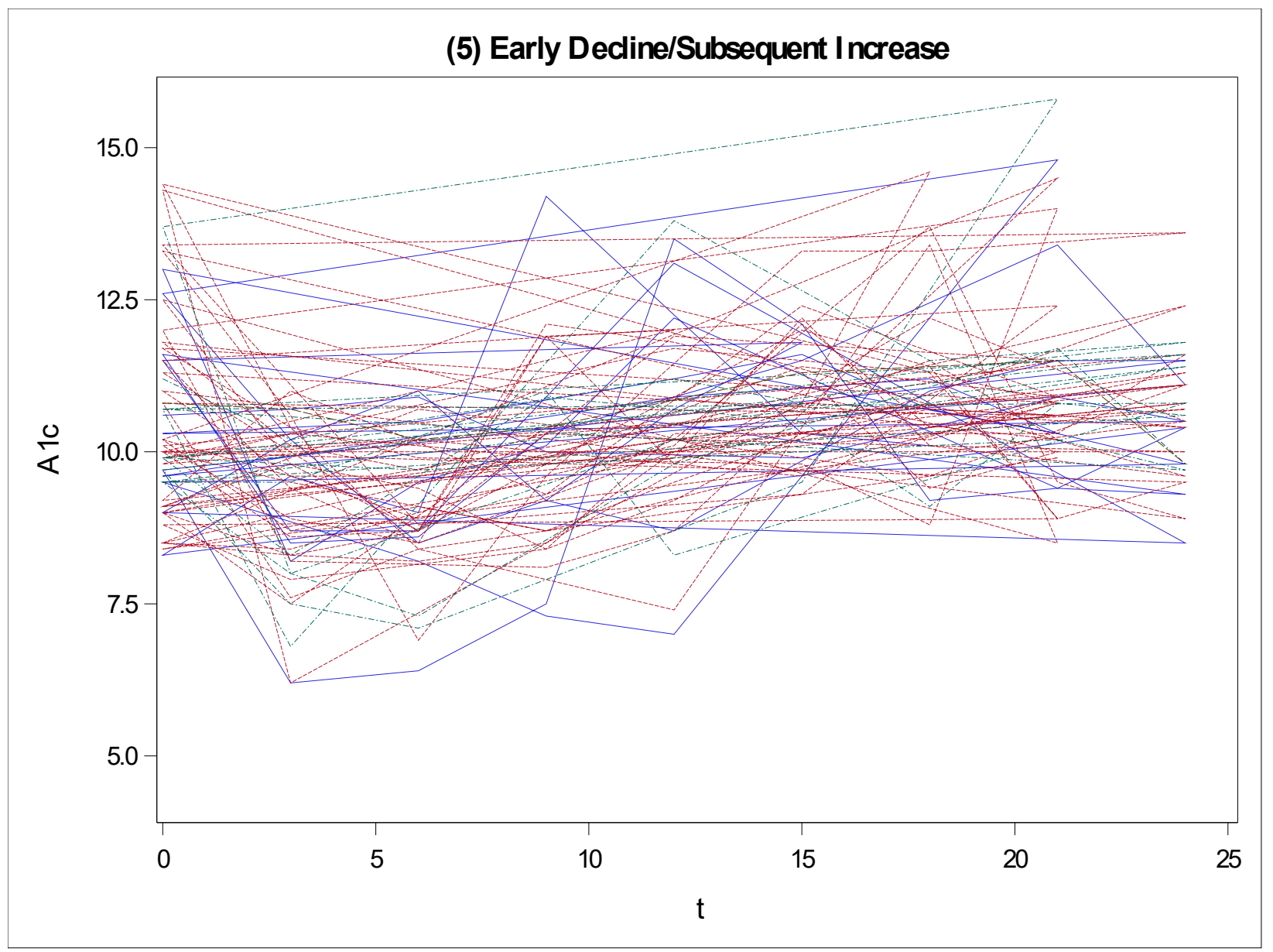

Figure 8: Early Decline / Subsequent Increase Example Trajectories 


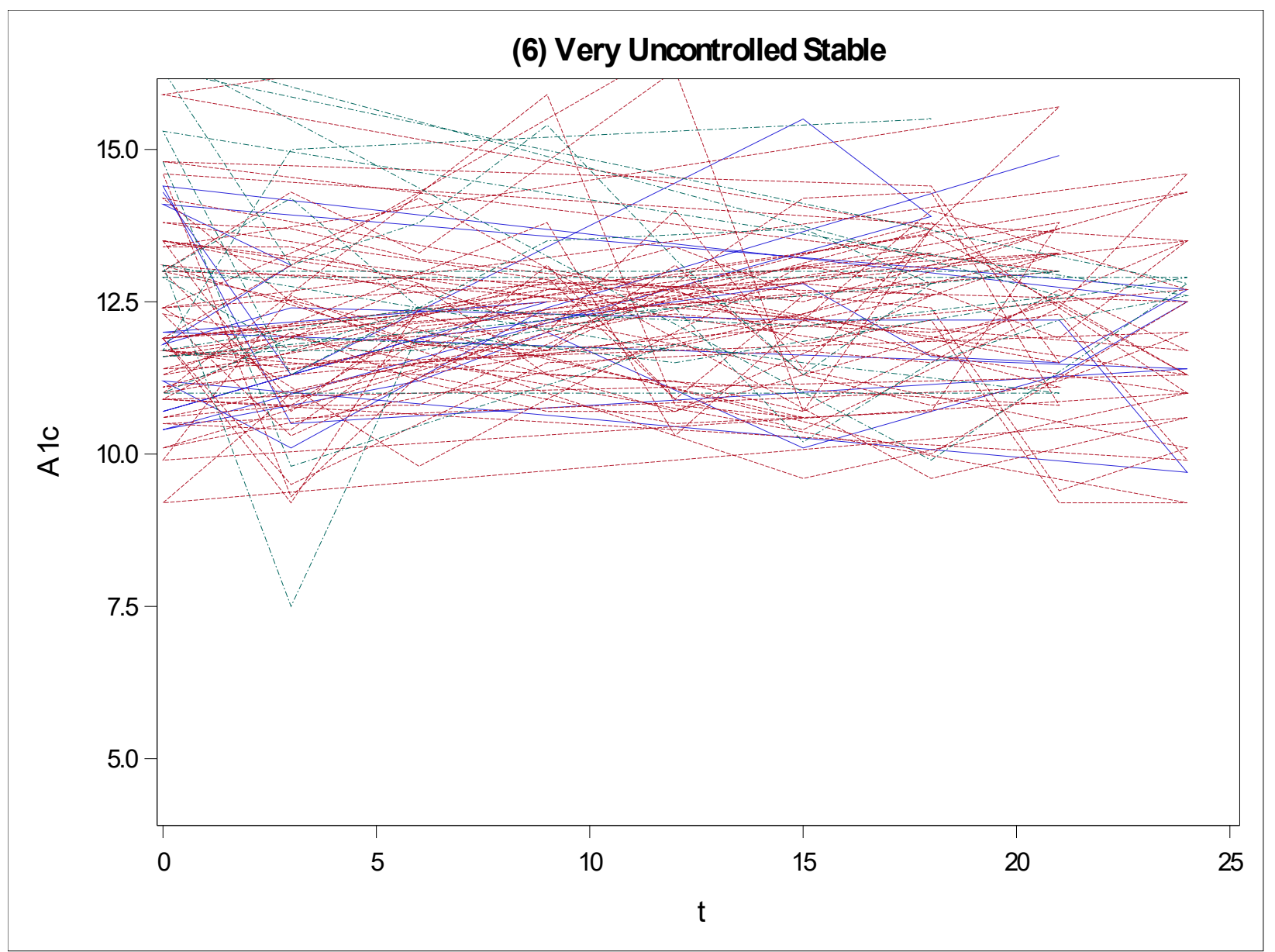

Figure 9: Very Uncontrolled Stable Example Trajectories 


\section{Appendix C: Full Regression Models}

\section{Predictors of Successful Trajectories}

The following table illustrates the full model of all predictors initially entered into the logistic regression model analysis prior to backwards selection of predictors.

Table 14: Full model predictors of successful trajectories

\begin{tabular}{|c|c|c|c|c|}
\hline \multirow{2}{*}{ Predictor } & \multirow{2}{*}{ OR } & \multicolumn{2}{|c|}{ 95\% Confidence Limits } & \\
\hline & & Lower & Upper & \\
\hline Intercept & - & - & | & 0.0659 \\
\hline Age & 1.011 & 1.003 & 1.018 & 0.0039 \\
\hline \multicolumn{4}{|c|}{ Duration of Pharmacy Follow-up (Ref: $<6$ Months) } & $<0.0001$ \\
\hline 6-12 Months & 0.571 & 0.475 & 0.686 & $<0.0001$ \\
\hline$\geq 12$ Months & 0.543 & 0.456 & 0.644 & $<0.0001$ \\
\hline Home Telehealth & 0.806 & 0.689 & 0.944 & 0.0073 \\
\hline \multicolumn{4}{|c|}{ Nutrition Clinic Follow-up (Ref: 0-1 visits) } & 0.0969 \\
\hline $2-5$ visits & 0.98 & 0.82 & 1.172 & 0.8253 \\
\hline $6+$ visits & 1.296 & 1.016 & 1.657 & 0.0374 \\
\hline \multicolumn{4}{|c|}{ Creatinine Clearance, baseline (Ref: $\geq 60 \mathrm{ml} / \mathrm{min}$ ) } & 0.0092 \\
\hline $30-59 \mathrm{ml} / \mathrm{min}$ & 0.868 & 0.7 & 1.078 & 0.1197 \\
\hline$<30 \mathrm{ml} / \mathrm{min}$ & 0.742 & 0.411 & 1.339 & 0.3191 \\
\hline Unknown & 0.627 & 0.469 & 0.837 & 0.0016 \\
\hline Test strips/day & 0.935 & 0.883 & 0.99 & 0.0206 \\
\hline \multicolumn{4}{|c|}{ Insulin at Baseline (Ref: None) } & 0.0065 \\
\hline Basal/Bolus & 0.769 & 0.631 & 0.937 & 0.0091 \\
\hline Basal only & 0.86 & 0.708 & 1.045 & 0.1281 \\
\hline Prandial only & 0.456 & 0.278 & 0.737 & 0.0015 \\
\hline U-500 & 0.61 & 0.149 & 2.341 & 0.4667 \\
\hline \multicolumn{4}{|l|}{ Medical Center (Ref: 1) } & $<0.0001$ \\
\hline 2 & 0.553 & 0.333 & 0.913 & 0.0212 \\
\hline 3 & 0.882 & 0.559 & 1.383 & 0.5863 \\
\hline 4 & 1.057 & 0.676 & 1.641 & 0.806 \\
\hline 5 & 0.596 & 0.365 & 0.968 & 0.0373 \\
\hline 6 & 0.896 & 0.55 & 1.452 & 0.657 \\
\hline 7 & 1.078 & 0.686 & 1.682 & 0.7417 \\
\hline 8 & 0.9 & 0.567 & 1.418 & 0.6506 \\
\hline Gender (Female) & 0.878 & 0.617 & 1.249 & 0.4676 \\
\hline \multicolumn{4}{|l|}{ Race (Ref = White): } & 0.5475 \\
\hline Black & 0.984 & 0.83 & 1.166 & 0.8488 \\
\hline Other & 0.849 & 0.634 & 1.138 & 0.273 \\
\hline Ratio of Phone Appts & 0.998 & 0.996 & 1.001 & 0.2878 \\
\hline Ratio of CVT Appts & 1.001 & 0.994 & 1.008 & 0.7861 \\
\hline Total DM Meds (BL) & 0.935 & 0.862 & 1.013 & 0.1016 \\
\hline Alcohol Use Disorder & 1.142 & 0.902 & 1.449 & 0.2729 \\
\hline Substance Use Disorder & 1.099 & 0.932 & 1.296 & 0.2634 \\
\hline Heart Failure & 0.826 & 0.675 & 1.012 & 0.0649 \\
\hline \multicolumn{5}{|c|}{ CVT $=$ Clinical Video Telehealth } \\
\hline \multicolumn{5}{|c|}{ Hosmer and Lemeshow Goodness of Fit Test $(p=0.2811)$} \\
\hline \multicolumn{5}{|c|}{ Predicted vs. Observed Percent Concordant $=62.3 \%$} \\
\hline
\end{tabular}




\section{Predictors of All-cause Hospital Admission}

The following table illustrates the full model of all predictors initially entered into the logistic regression model analysis prior to backwards selection of predictors.

Table 15: Full model predictors of all-cause hospital admission

\begin{tabular}{|c|c|c|c|c|}
\hline \multirow{2}{*}{ Predictor (Any Admission) } & \multirow{2}{*}{ OR } & \multicolumn{2}{|c|}{ 95\% Confidence Limits } & \multirow{2}{*}{$p$} \\
\hline & & Lower & Upper & \\
\hline Intercept & - & - & - & $<0.0001$ \\
\hline Age & 1.016 & 1.007 & 1.025 & 0.0003 \\
\hline Gender (Female) & 0.943 & 0.609 & 1.42 & 0.7846 \\
\hline Race (Ref: White) & & & & 0.3625 \\
\hline Black of African American & 1.019 & 0.838 & 1.235 & 0.8512 \\
\hline Other & 0.782 & 0.546 & 1.1 & 0.1677 \\
\hline Heart Failure & 2.674 & 2.18 & 3.28 & $<0.0001$ \\
\hline Alcohol Use Disorder & 2.06 & 1.612 & 2.628 & $<0.0001$ \\
\hline Substance Use Disorder & 1.628 & 1.36 & 1.945 & $<0.0001$ \\
\hline \multicolumn{2}{|c|}{ Creatinine Clearance, baseline (Ref: $\geq 60 \mathrm{ml} / \mathrm{min}$ ) } & & & $<0.0001$ \\
\hline $30-59 \mathrm{ml} / \mathrm{min}$ & 1.47 & 1.169 & 1.846 & 0.0009 \\
\hline$<30 \mathrm{ml} / \mathrm{min}$ & 2.831 & 1.558 & 5.177 & 0.0006 \\
\hline Unknown & 0.761 & 0.522 & 1.087 & 0.1441 \\
\hline Baseline HbA1c & 1.111 & 1.042 & 1.185 & 0.0013 \\
\hline \multicolumn{2}{|c|}{ Trajectory Group (Ref: Moderate Early Decline) } & & & 0.0294 \\
\hline Large Early Decline & 0.65 & 0.42 & 0.996 & 0.0499 \\
\hline High Gradually Decreasing & 1.224 & 0.893 & 1.67 & 0.2055 \\
\hline Uncontrolled Stable & 0.988 & 0.833 & 1.17 & 0.8866 \\
\hline Early Decline/Subsequent Increase & 1.242 & 0.911 & 1.682 & 0.1647 \\
\hline Very Uncontrolled Stable & 1.131 & 0.72 & 1.758 & 0.5872 \\
\hline \multicolumn{2}{|l|}{ Medical Center (Ref: 1) } & & & 0.0032 \\
\hline 2 & 0.779 & 0.439 & 1.409 & 0.399 \\
\hline 3 & 1.083 & 0.653 & 1.853 & 0.7643 \\
\hline 4 & 1.176 & 0.718 & 1.993 & 0.5316 \\
\hline 5 & 1.128 & 0.661 & 1.979 & 0.6656 \\
\hline 6 & 1.191 & 0.697 & 2.093 & 0.531 \\
\hline 7 & 0.807 & 0.479 & 1.399 & 0.4296 \\
\hline 8 & 0.807 & 0.479 & 1.399 & 0.4318 \\
\hline \multicolumn{4}{|c|}{ Hosmer and Lemeshow Goodness of Fit Test $(p=0.4351)$} & \\
\hline
\end{tabular}




\section{Predictors of Diabetes-related Hospital Admission}

The following table illustrates the full model of all predictors initially entered into the logistic regression model analysis prior to backwards selection of predictors.

Table 16: Full model predictors of diabetes-related hospital admission

\begin{tabular}{|c|c|c|c|c|}
\hline \multirow{2}{*}{ Predictor (DM Admission) } & \multirow{2}{*}{ OR } & \multicolumn{2}{|c|}{ 95\% Confidence Limits } & \multirow{2}{*}{$\mathrm{p}$} \\
\hline & & Lower & Upper & \\
\hline Intercept & - & - & - & $<0.0001$ \\
\hline Age & 1.011 & 0.993 & 1.031 & 0.2318 \\
\hline Gender (Female) & 1.296 & 0.531 & 2.7 & 0.5257 \\
\hline \multicolumn{2}{|l|}{ Race (Ref: White) } & & & 0.5727 \\
\hline Black of African American & 1.111 & 0.74 & 1.644 & 0.6037 \\
\hline Other & 0.706 & 0.288 & 1.478 & 0.3989 \\
\hline Heart Failure & 1.085 & 0.667 & 1.698 & 0.7312 \\
\hline Alcohol Use Disorder & 2.143 & 1.346 & 3.328 & 0.0009 \\
\hline Substance Use Disorder & 1.893 & 1.311 & 2.706 & 0.0005 \\
\hline \multicolumn{2}{|c|}{ Creatinine Clearance, baseline (Ref: $\geq 60 \mathrm{ml} / \mathrm{min}$ ) } & & & 0.0076 \\
\hline $30-59 \mathrm{ml} / \mathrm{min}$ & 1.79 & 1.103 & 2.836 & 0.0154 \\
\hline$<30 \mathrm{ml} / \mathrm{min}$ & 4.199 & 1.361 & 10.638 & 0.0053 \\
\hline Unknown & 1.296 & 0.531 & 2.7 & 0.7956 \\
\hline Baseline HbA1c & 1.079 & 0.947 & 1.226 & 0.2486 \\
\hline \multicolumn{2}{|c|}{ Trajectory Group (Ref: Moderate Early Decline) } & & & $<0.0001$ \\
\hline Large Early Decline & 0.659 & 0.198 & 1.861 & 0.4589 \\
\hline High Gradually Decreasing & 2.931 & 1.542 & 5.479 & 0.0009 \\
\hline Uncontrolled Stable & 1.803 & 1.191 & 2.759 & 0.0058 \\
\hline Early Decline/Subsequent Increase & 3.549 & 1.956 & 6.288 & $<0.0001$ \\
\hline Very Uncontrolled Stable & 5.213 & 2.468 & 10.752 & $<0.0001$ \\
\hline \multicolumn{2}{|l|}{ Medical Center (Ref: 1) } & & & 0.3386 \\
\hline 2 & 0.806 & 0.215 & 3.853 & 0.7617 \\
\hline 3 & 1.18 & 0.395 & 5.085 & 0.7927 \\
\hline 4 & 0.893 & 0.301 & 3.84 & 0.8575 \\
\hline 5 & 1.451 & 0.462 & 6.41 & 0.5657 \\
\hline 6 & 0.466 & 0.111 & 2.335 & 0.3074 \\
\hline 7 & 1.268 & 0.446 & 5.335 & 0.6982 \\
\hline 8 & 0.965 & 0.31 & 4.239 & 0.9554 \\
\hline \multicolumn{4}{|c|}{ Hosmer and Lemeshow Goodness of Fit Test $(p=0.194)$} & \\
\hline Predicted vs. Observed Percent & ordant $=7$ & $70.2 \%$ & & \\
\hline
\end{tabular}

